\title{
GLASS TRANSITION IN SYSTEMS WITHOUT STATIC CORRELATIONS: A MICROSCOPIC THEORY
}

\author{
R. Schilling ${ }^{1}$ and G. Szamel ${ }^{1,2}$ \\ ${ }^{1}$ Institut für Physik, Johannes Gutenberg-Universität Mainz, D-55099 Mainz, \\ Germany \\ ${ }^{2}$ Department of Chemistry, Colorado State University, Ft. Collins, CO 80523, USA
}

\begin{abstract}
We present a first step toward a microscopic theory for the glass transition in systems with trivial static correlations. As an example we have chosen $N$ infinitely thin hard rods with length $L$, fixed with their centers on a periodic lattice with lattice constant $a$. Starting from the $N$-rod Smoluchowski equation we derive a coupled set of equations for fluctuations of reduced $k$-rod densities. We approximate the influence of the surrounding rods onto the dynamics of a pair of rods by introduction of an effective rotational diffusion tensor $\mathbf{D}\left(\Omega_{1}, \Omega_{2}\right)$ and in this way we obtain a selfconsistent equation for $\mathbf{D}$. This equation exhibits a feedback mechanism leading to a slowing down of the relaxation. It involves as an input the Laplace transform $v_{0}(l / r)$ at $z=0, l=L / a$, of a torque-torque correlator of an isolated pair of rods with distance $R=a r$. Our equation predicts the existence of a continuous ergodicity-breaking transition at a critical length $l_{c}=L_{c} / a$. To estimate the critical length we perform an approximate analytical calculation of $v_{0}(l / r)$ based on a variational approach and obtain $l_{c}^{\mathrm{var}} \cong 5.68,4.84$ and 3.96 for an sc, bcc and fcc lattice. We also evaluate $v_{0}(l / r)$ numerically exactly from a two-rod simulation. The latter calculation leads to $l_{c}^{\text {num }} \cong 3.45,2.78$ and 2.20 for the corresponding lattices. Close to $l_{c}$ the rotational diffusion constant decreases as $D(l) \sim\left(l_{c}-l\right)^{\gamma}$ with $\gamma=1$ and a diverging time scale $t_{\epsilon} \sim\left|l_{c}-l\right|^{-\delta}, \delta=2$, appears. On this time scale the $t$ - and $l$-dependence of the 1-rod density is determined by a master function depending only on $t / t_{\epsilon}$. In contrast to present microscopic theories our approach predicts a glass transition despite the absence of any static correlations.
\end{abstract}

PACS numbers: 61.20. Lc, 61.43. Fs, 64.70. Pf

Submitted to: J. Phys.: Condens. Matter 


\section{INTRODUCTION}

In the last two decades huge theoretical efforts were made in order to describe the glass transition on a microscopic level in systems without quenched disorder. Microscopic means that, e.g., the glass transition temperature and the dynamical properties in its vicinity can be calculated from equations which use as an input the knowledge of the interactions between microscopic constituents of the system (atoms or molecules; hereafter we use the term particles). Most of this activity has been devoted to structural glasses [1, 2, 3, ㅂ. Although experiments [5, 6, 7, 8] have demonstrated that plastic crystals can exhibit an orientational glass transition which has many features in common with the structural glass transition there seems to be no analytical theory for such systems. 团 On the other hand Molecular Dynamics (MD) simulations [11, 12, 13] have confirmed the similarity between glassy behavior of plastic crystals and that of supercooled liquids.

The first successful microscopic theoretical approach to glassy dynamics in supercooled liquids came from so-called mode coupling theory (MCT) [1, 2]. This theory was first suggested and mainly worked out in details by Götze and his coworkers. For reviews the reader may consult refs. [14, 15, 16]. MCT derives a closed set of equations for time-dependent correlators, like the intermediate scattering function $S(q, t)$. This set uses as an input the corresponding static correlations, e.g. $S(q)$, which depend only on thermodynamic variables like temperature $T$, density $n$, etc. The static correlations can be calculated from the microscopic interactions by use of either analytical or numerical tools [17]. By decreasing $T$ (or increasing $n$ ) the peaks of $S(q)$ grow. If their magnitude becomes large enough a transition from an ergodic to a non-ergodic phase occurs at a critical temperature $T_{c}$ (or density $n_{c}$ ). This dynamic transition is interpreted as an ideal glass transition. MCT makes several nontrivial predictions for, e.g., time dependence of correlators [14, 15, 16] which have been successfully tested (see [18, 19, 20, 21] for reviews).

The second microscopic theory of the glass transition has been developed by Mézard and Parisi [3]. Their first principle theory combines the use of the replica idea known from the spin glass theory and of the liquid state theory techniques. It is related to an earlier density functional approach [⿴囗十 microscopic Hamiltonian for $m$ identical replicas of the system to which a symmetry breaking field is added. The free energy of the replicated system is then calculated and the configurational entropy of the original system is extracted from it. The theory predicts the existence of a critical temperature $T_{f}$ (which is below $T_{c}$ of $\mathrm{MCT}$ ) at which the configurational entropy vanishes. This is then interpreted as an ideal static glass transition.

Besides these two microscopic theories, let us mention one other attempt to describe the glass transition that does not resort to microscopic potentials: based on several

$\dagger$ The microscopic theory developed by Michel and coworkers [9, 10] is valid for the orientational glass transition of mixed crystals, i.e. crystals with quenched disorder. 
qualitative assumptions Edwards and Vilgis 222 obtained the dependence of the selfdiffusion constant $D$ for a system of hard rods on physical control parameter $x$, like, e.g., concentration, diameter, or length of the rods. A dynamical glass transition occurs when $D$ becomes zero. A mean field treatment results in $D \sim x_{c}-x$ and an improved approach leads to the Vogel-Fulcher law $D \sim \exp \left[A /\left(x_{c}-x\right)\right]$. Close to the critical point $x_{c}$ a power law dependence of $D$ on $\left(x_{c}-x\right)$ is found with a non-trivial exponent $\gamma=7 / 6$.

Both above described microscopic theories have at least one feature in common: non-trivial equilibrium correlations. These correlations are of primary importance for both theories. For example, MCT requires that the vertices which enter into the selfconsistent equations for the correlation functions must depend on at least one control parameter. Recently, it has been shown that a colloidal suspension with hard-core plus attractive Yukawa potential can undergo a gel transition [23] in a special limit: $\phi \rightarrow 0$ and $K \rightarrow \infty$ such that $\Gamma=K^{2} \phi / b=$ const. Here $\phi$ is the volume fraction, $K$ the interaction strength and $b$ a measure of the inverse range of interaction. In that limit the direct correlation function $c(q)$ is proportional to $K$ and becomes independent of $\phi$ [24]. This implies that the static correlations vanish, i.e. $S(q) \rightarrow 1$. But since the vertices are proportional to $\phi$ and involve a bilinear product of $c(q)$ they become proportional to $\Gamma$. Accordingly, despite the vanishing of the static correlations there is still an ergodicity breaking transition driven by the increase of $\Gamma$ 223, 24, 25]. Of course, this type of behavior is not generic in the $\phi-K$ phase space. In addition, whether the mode coupling approximation remains reasonable must be still checked.

One may now ask the following question: Can there be a generic glass transition if and only if there exist nontrivial static correlations? To find an answer let us consider a liquid of hard rods. This system was used as a paradigm in ref. [22]. If the rods thickness $d$ is finite the static correlations are non-trivial and a glass transition will be predicted by either theory. However, in the limit of infinitely thin hard rods, i. e. $d=0$, with finite length $L$ and finite concentration $c$, the static properties become trivial. Note that in this limit there is no transition to a nematic phase. Also, according to either of the two microscopic theories there is no glass transition! In the case of MCT the vertices vanish. The absence of a glass transition is also consistent with a microscopic theory worked out by one of the authors and his coworkers [26, 27. There, it has been shown that for infinitely thin hard rods with randomly frozen orientations translational diffusion never ceases if the diffusion constant along the rod's direction is nonzero.

Now suppose we fix the infinitely thin hard rods with their centers on the sites of a three-dimensional periodic lattice. Such a model has already been suggested earlier [22, 28]. It can be used to describe the steric hindrance of the orientational degrees of freedom in plastic crystals [6, 7, 8, 11, 12, 13]. Again, both MCT and the replica theory exclude a glass transition, due to the lack of static correlations. The fact that MCT in its present form does not predict a glass transition was already stressed in ref. [29]. Nevertheless, computer simulations [29, 30] showed glassy behavior and suggested a 
dynamical ergodicity-breaking transition at a critical length $L_{c}$. 目 This simple example makes clear that non-trivial static behavior is not necessary for a glass transition. It is the main goal of our paper to present a microscopic approach to the glass transition for systems without static correlations.

A short account of our approach for the case of a simple cubic lattice was given in ref. 31]. In the present paper we will discuss our model and the theoretical framework in more detail and will extend the results to bcc and fcc lattices. Furthermore, we will investigate the type of glass transition and the long time dynamics in its vicinity.

The outline of our paper is as follows. In the next section we will present and discuss the model. The third section contains the analytical theory used to describe the glass transition. The results are given in the fourth section and the final section includes a summary and some conclusions.

\section{MODEL}

We consider $N$ hard, infinitely thin rods (i.e. diameter $d=0$ ) with length $L$ and with their centers fixed at the lattice sites of a periodic lattice with lattice constant $a$. The dimensionless control parameter is the reduced rod length, $l=L / a$. In the following we will restrict ourselves to a simple cubic lattice. Its unit cell is depicted in Fig. 11. All qualitative conclusions which will be drawn in this paper remain valid for all periodic lattices.

It is obvious that for $l \leq 1$ each rod can rotate freely: there is no steric hindrance. For $l$ above one, collisions between a rod and adjacent ones appear. If $1 \leq l \leq \sqrt{3}$ a given rod interacts only with the six nearest neighbors. Further increase of $l$ will introduce collisions between next-, third-, fourth-, etc. nearest neighbors, generating a strong increase of steric hindrance. Since we have chosen infinitely thin rods there is no equilibrium transition to an orientationally ordered phase. On the other hand, due to the rise of steric hindrance with $l$ we expect an orientational glass transition as detected in ref. [29] and [30]. Although steric hindrance already exists for $l>1$, it seems that no ergodicity-breaking transition can occur for $l<2$. Let us consider the following simple argument. If $l>2$ then the nearest neighbor rods of a tagged rod (rod 1) can "overlap" with, e.g., the top of the tagged rod (see Fig. 2). This induces a "cage" for the tagged rod. Note that this argument is not rigorous, because for $l<2$ there might exist other configurations similar to the one shown in Fig. 2 which could block the tagged rod's rotation. But the value $l=2$ also plays a role from a different point of view. Consider two neighboring rods with their centers along the $z$-axis. Their orientations can be described by $\Omega_{i}=\left(\theta_{i}, \phi_{i}\right)$ and $\Omega_{j}=\left(\theta_{j}, \phi_{j}\right)$. At a collision it is either $\phi_{j}=\phi_{i}^{ \pm}$(see Fig. 3 a) or $\phi_{j}=\phi_{i}^{ \pm}+\pi$ (see Fig. 3 b), for fixed $\theta_{i}, \theta_{j}$ and $\phi_{i}$. The notation $\phi_{i}^{+}$and $\phi_{i}^{-}$means that $\operatorname{rod} j$ is behind and in front of $\operatorname{rod} i$, respectively (see Figure 3). Now, it is easy to prove that for arbitrary $\theta_{i}$ and $\theta_{j}$ there exists only one $\ddagger$ Not that different cubic lattices were used in refs. [29] and [30; moreover the authors of ref. 30] fixed the rods with one of their endpoints at the lattice sites. 
contact (either at $\phi_{i}^{ \pm}$or at $\phi_{i}^{ \pm}+\pi$ ) for $l<2$ (see Fig. 3 a,b). In that case $\phi_{j}$ can be varied freely by $2 \pi$. But for $l>2$ there is a finite range for both angles $\theta_{i}$ and $\theta_{j}$ such that two contacts (at $\phi_{i}^{ \pm}$and $\phi_{i}^{ \pm}+\pi$ ) exist (see Fig. 3 c). Therefore the "phase space" $[0,2 \pi]$ for $\phi_{j}-\phi_{i}$ is decomposed into two "ergodic" components $[0, \pi]$ and $[\pi, 2 \pi]$ for $\theta_{i}$ and $\theta_{j}$ kept fixed. Transitions between both components are forbidden. If the rods were fixed at the lattice sites with one of their end points (like in ref. [30]) then there is only one contact, for all $l>1$.

The condition for a collision can be quantified as follows 32. Let $\mathbf{u}_{i}$ and $\mathbf{u}_{j}$ be the unit vectors along rod $i$ and $j$ and $\mathbf{r}_{i j}$ the vector connecting the center of rod $j$ with that of $\operatorname{rod} i$ (see Fig. (4). $\mathbf{u}_{i}$ and $\mathbf{u}_{j}$ define a plane. Let $\mathbf{r}_{i j}^{\perp}$ be the component of $\mathbf{r}_{i j}$ perpendicular to that plane and $r_{i j}^{\perp}=\left|\mathbf{r}_{i j}^{\perp}\right|$. Denote the distance of the point of contact to the center of rod $i$ and $j$ by, respectively, $s_{i j}$ and $s_{j i}$ (Fig. 4 ). Then a collision occurs if:

$$
r_{i j}^{\perp}=0^{+} \quad, \quad\left|s_{i j}\right|<L / 2 \quad, \quad\left|s_{j i}\right|<L / 2
$$

where $0^{+}$stands for limit $d \rightarrow 0$. Simple algebra yields:

$$
\begin{aligned}
& s_{i j}=-\left[\left(\mathbf{u}_{i} \cdot \mathbf{r}_{i j}\right)-\left(\mathbf{u}_{i} \cdot \mathbf{u}_{j}\right)\left(\mathbf{u}_{j} \cdot \mathbf{r}_{i j}\right)\right] /\left[1-\left(\mathbf{u}_{i} \cdot \mathbf{u}_{j}\right)^{2}\right] \\
& s_{j i}=\left[\left(\mathbf{u}_{j} \cdot \mathbf{r}_{i j}\right)-\left(\mathbf{u}_{i} \cdot \mathbf{u}_{j}\right)\left(\mathbf{u}_{i} \cdot \mathbf{r}_{i j}\right)\right] /\left[1-\left(\mathbf{u}_{i} \cdot \mathbf{u}_{j}\right)^{2}\right]
\end{aligned}
$$

Note (i) that $s_{i j}$ and $s_{j i}$ can be negative; in that case the contact points into the direction of $-\mathbf{u}_{i}$ and $-\mathbf{u}_{j}$, respectively and (ii) that $\mathbf{r}_{i j}$ is time-independent and is always equal to a lattice vector $\mathbf{R}_{i j}=\mathbf{R}_{i}-\mathbf{R}_{j}$.

The configurational part of the partition function is given by

$$
Z_{c}(T, N ; l)=\int \prod_{i=1}^{N} d \Omega_{i} e^{-\beta V\left(\Omega_{1}, \cdots, \Omega_{N}\right)}=(4 \pi)^{N} \quad, \quad \beta=\left(k_{B} T\right)^{-1}
$$

since the hard rod interaction potential $V\left(\Omega_{1}, \cdots, \Omega_{N}\right)$ vanishes almost everywhere. The corresponding contribution $-k_{B} T N \ln 4 \pi$ to the free energy is analytic in temperature $T$ and does not depend on $l$. Hence, there is no equilibrium phase transition. The equilibrium pair distribution function $g_{i j}^{(2)}\left(\Omega_{i}, \Omega_{j}\right)$ is related to the probability to find a rod at $\mathbf{R}_{i}$ and $\mathbf{R}_{j}\left(\neq \mathbf{R}_{i}\right)$ with orientations $\Omega_{i}$ and $\Omega_{j}$, respectively. It is given by:

$$
g_{i j}^{(2)}\left(\Omega_{i}, \Omega_{j}\right)=1-\theta\left(0^{+}-r_{i j}^{\perp}\right) \theta\left(\frac{L}{2}-\left|s_{i j}\right|\right) \theta\left(\frac{L}{2}-\left|s_{j i}\right|\right)
$$

where the Heaviside functions are nonzero at a collision, which happens if conditions Eq. (1) are fulfilled. Since a collision is non-generic, $g_{i j}^{(2)}\left(\Omega_{i}, \Omega_{j}\right)=1$ for almost all $\Omega_{i}$ and $\Omega_{j}$. Hence there are no static correlations, except on a set of measure zero. 


\section{KINETIC THEORY}

In this section we will describe the dynamics for our model and the approximations leading to a closed set of equations. We assume that the microscopic $N$-rod dynamics of the system is Brownian rather than Newtonian. Previous studies of systems with nontrivial static correlations indicated that glassy dynamics should not depend on that choice [33, 34].

The starting point of the theory is the so-called generalized Smoluchowski equation for the $N$-rod probability density $P_{N}\left(\Omega_{1}, \cdots, \Omega_{N} ; t\right)$ [32]

$$
\frac{\partial}{\partial t} P_{N}\left(\Omega_{1}, \cdots, \Omega_{N} ; t\right)=D_{0} \sum_{n=1}^{N} \nabla_{n} \cdot\left[\nabla_{n}-\mathbf{T}_{n}\left(\Omega_{1}, \cdots, \Omega_{N}\right)\right] P_{N}\left(\Omega_{1}, \cdots, \Omega_{N} ; t\right)(6)
$$

where $D_{0}$ is the bare rotational diffusion constant, $\nabla_{n} \equiv \nabla_{\Omega_{n}} \equiv \mathbf{u}_{n} \times \nabla_{\mathbf{u}_{n}}$, and

$$
\mathbf{T}_{n}\left(\Omega_{1}, \cdots, \Omega_{N}\right)=\sum_{j \neq n} \mathbf{T}_{j n}\left(\Omega_{j}, \Omega_{n}\right) .
$$

$\mathbf{T}_{i j}\left(\Omega_{i}, \Omega_{j}\right)$ describes the singular torque when two rods at sites $i$ and $j$ with vector distance $\mathbf{r}_{i j}$ collide:

$$
\mathbf{T}_{i j}\left(\Omega_{i}, \Omega_{j}\right)=s_{i j}\left(\mathbf{u}_{i} \times \widehat{\mathbf{r}}_{i j}^{\perp}\right) \delta\left(r_{i j}^{\perp}-0^{+}\right) \theta\left(\frac{L}{2}-\left|s_{i j}\right|\right) \theta\left(\frac{L}{2}-\left|s_{j i}\right|\right)
$$

where $\widehat{\mathbf{r}}_{i j}^{\perp}=\mathbf{r}_{i j}^{\perp} / r_{i j}^{\perp}$. Using Eq. (5) it is easy to prove that

$$
\nabla_{i} g_{i j}^{(2)}\left(\Omega_{i}, \Omega_{j}\right)=\mathbf{T}_{i j}\left(\Omega_{i}, \Omega_{j}\right) g_{i j}^{(2)}\left(\Omega_{i}, \Omega_{j}\right) .
$$

Eq. (9) describes the noncrossability condition. Note that $\mathbf{T}_{i j}\left(\Omega_{i}, \Omega_{j}\right)$ differs slightly from $\mathbf{T}_{i j}\left(\Omega_{i}, \Omega_{j}\right)$ used in ref. [32] since there $\nabla_{u_{i}}$ was used instead of $\nabla_{\Omega_{i}}$. Also, a similar generalized Smoluchowski equation had been used before to describe a liquid of infinitely thin hard rods with randomly frozen orientations [26, 27].

In a next step we present equations of motion for the reduced $k$-rod density

$$
\rho_{n_{1} \cdots n_{k}}^{(k)}\left(\Omega_{1}, \cdots, \Omega_{k} ; t\right)=\int \prod_{n \neq n_{1}, \cdots, n_{k}} d \Omega_{n} P_{N}\left(\Omega_{1}, \cdots, \Omega_{N} ; t\right) .
$$

These equations form an infinite, coupled hierarchy [35] of the following form

$$
\begin{aligned}
& \frac{\partial}{\partial t} \rho_{n_{1} \cdots n_{k}}^{(k)}\left(\Omega_{1}, \cdots, \Omega_{k} ; t\right)=\mathcal{L}^{(k)} \rho_{n_{1} \cdots n_{k}}^{(k)}\left(\Omega_{1}, \cdots, \Omega_{k} ; t\right)- \\
& -D_{0} \sum_{n \in I_{k}} \sum_{n_{k+1} \notin I_{k}} \nabla_{n} \cdot \int d \Omega_{k+1} \mathbf{T}_{n n_{k+1}}\left(\Omega_{n}, \Omega_{k+1}\right) \\
& \times \rho_{n_{1} \cdots n_{k+1}}^{(k+1)}\left(\Omega_{1}, \cdots, \Omega_{k+1} ; t\right)
\end{aligned}
$$

with the $k$-rod Smoluchowski operator,

$$
\mathcal{L}^{(k)}=D_{0} \sum_{n \in I_{k}} \nabla_{n} \cdot\left[\nabla_{n}-\sum_{\substack{n^{\prime} \in I_{k} \\ n^{\prime}(\neq n)}} \mathbf{T}_{n n^{\prime}}\left(\Omega_{n}, \Omega_{n^{\prime}}\right)\right]
$$


and $I_{k}=\left\{n_{1}, \cdots, n_{k}\right\}$. As initial conditions we choose:

$$
\rho_{n_{1} \cdots n_{k}}^{(k)}\left(\Omega_{1}, \cdots, \Omega_{k} ; 0\right)=\frac{1}{(4 \pi)^{k-1}} \delta\left(\Omega_{1} \mid \Omega_{0}\right) g_{n_{1} \cdots n_{k}}^{(k)}\left(\Omega_{1}, \cdots, \Omega_{k}\right),
$$

where $\delta\left(\Omega \mid \Omega^{\prime}\right)=\sin \theta \delta\left(\theta-\theta^{\prime}\right) \delta\left(\phi-\phi^{\prime}\right)$. Here, $g_{n_{1} \cdots n_{k}}^{(k)}\left(\Omega_{1}, \cdots, \Omega_{k}\right)$ is the equilibrium $k$-rod distribution function which is equal to one almost everywhere.

To proceed we introduce the fluctuations of the $k$-rod density for $k \geq 2$

$$
\begin{aligned}
& \delta \rho_{n_{1} \cdots n_{k}}^{(k)}\left(\Omega_{1}, \cdots, \Omega_{k} ; t\right)=\rho_{n_{1} \cdots n_{k}}^{(k)}\left(\Omega_{1}, \cdots, \Omega_{k} ; t\right) \\
& -\frac{1}{(4 \pi)^{k-1}} g_{n_{1} \cdots n_{k}}^{(k)}\left(\Omega_{1}, \cdots, \Omega_{k}\right) \rho_{n_{1}}^{(1)}\left(\Omega_{1} ; t\right) .
\end{aligned}
$$

These fluctuations vanish in equilibrium and for $t=0$.

Substituting $\rho^{(k)}$ from Eq. (14) into Eq. (11) and taking into account that the equilibrium $k$-rod rotational current density vanishes we obtain the following equation

$$
\frac{\partial}{\partial t} \rho_{n_{1}}^{(1)}\left(\Omega_{1} ; t\right)+\nabla_{1} \cdot \mathbf{j}_{n_{1}}^{(1)}\left(\Omega_{1} ; t\right)=0
$$

where the 1-rod rotational current density reads

$$
\begin{aligned}
& \mathbf{j}_{n_{1}}^{(1)}\left(\Omega_{1} ; t\right)=-D_{0}\left\{\nabla_{1} \rho_{n_{1}}^{(1)}\left(\Omega_{1} ; t\right)-\right. \\
& \left.\sum_{n_{2} \neq n_{1}} \int d \Omega_{2} \mathbf{T}_{n_{1} n_{2}}\left(\Omega_{1}, \Omega_{2}\right) \delta \rho_{n_{1} n_{2}}^{(2)}\left(\Omega_{1}, \Omega_{2} ; t\right)\right\} .
\end{aligned}
$$

At the next level, $k=2$, it follows by use of Eq. (9)

$$
\begin{aligned}
& \frac{\partial}{\partial t} \delta \rho_{n_{1} n_{2}}^{(2)}\left(\Omega_{1}, \Omega_{2} ; t\right)=-\frac{1}{4 \pi} \mathbf{T}_{n_{1}, n_{2}}\left(\Omega_{1}, \Omega_{2}\right) g_{n_{1} n_{2}}^{(2)}\left(\Omega_{1}, \Omega_{2}\right) \cdot \mathbf{j}_{n_{1}}^{(1)}\left(\Omega_{1} ; t\right)+ \\
& +D_{0}\left\{\nabla_{1} \cdot\left[\nabla_{1}-\mathbf{T}_{n_{1} n_{2}}\left(\Omega_{1}, \Omega_{2}\right)\right]+(1 \leftrightarrow 2)\right\} \delta \rho_{n_{1} n_{2}}^{(2)}\left(\Omega_{1}, \Omega_{2} ; t\right)+ \\
& +A_{n_{1} n_{2}}^{(2)}\left(\Omega_{1}, \Omega_{2} ; t\right)
\end{aligned}
$$

where

$$
\begin{aligned}
& A_{n_{1} n_{2}}^{(2)}\left(\Omega_{1}, \Omega_{2} ; t\right)= \\
& D_{0} \nabla_{1} \cdot\left[g_{n_{1} n_{2}}^{(2)}\left(\Omega_{1}, \Omega_{2}\right) \sum_{n_{3}} \frac{1}{4 \pi} \int d \Omega_{3} \mathbf{T}_{n_{1} n_{3}}\left(\Omega_{1}, \Omega_{3}\right) \delta \rho_{n_{1} n_{3}}^{(2)}\left(\Omega_{1}, \Omega_{3} ; t\right)\right] \\
& -D_{0} \sum_{\nu=1}^{2} \nabla_{\nu} \cdot \sum_{n_{3}} \int d \Omega_{3} \mathbf{T}_{n_{\nu} n_{3}}\left(\Omega_{\nu}, \Omega_{3}\right) \delta \rho_{n_{1} n_{2} n_{3}}^{(3)}\left(\Omega_{1}, \Omega_{2}, \Omega_{3} ; t\right) .
\end{aligned}
$$

Let us interpret these equations: Eq. (15) is the continuity equation. The corresponding 1-rod current density (Eq. (16)) consists of two parts. The first describes the contribution from the free Brownian dynamics of a tagged rod at site $n_{1}$ and the 
second is due to the interaction of the tagged rod with a second rod at side $n_{2}$. The first line on the r.h.s. of Eq. (17) originates from the last term on the r.h.s. of Eq. (14), its second line describes Brownian dynamics of an isolated pair of rods at sites $n_{1}$ and $n_{2}$, and the last line contains the influence of a third rod at $n_{3}$ on the rods at $n_{1}$ and $n_{2}$. In order to close this set of equations we follow the strategy of refs. [26, 27] and approximate the influence of a third rod by introducing an effective, nonlocal (in angular space and time) diffusion tensor $\mathbf{D}^{\text {eff }}\left(\Omega, \Omega^{\prime} ; t\right)$ and simultaneously neglecting $A_{n_{1} n_{2}}^{(2)}$ :

$$
\begin{aligned}
& \left\{D_{0} \nabla_{1} \cdot\left[\nabla_{1}-\mathbf{T}_{n_{1} n_{2}}\left(\Omega_{1}, \Omega_{2}\right)\right]+(1 \leftrightarrow 2)\right\} \delta \rho_{n_{1} n_{2}}^{(2)}\left(\Omega_{1}, \Omega_{2} ; t\right) \rightarrow \\
& \left\{\nabla_{1} \cdot \int_{0}^{t} d t^{\prime} \int d \Omega_{1}^{\prime} \mathbf{D}^{\mathrm{eff}}\left(\Omega_{1}, \Omega_{1}^{\prime} ; t-t^{\prime}\right) \cdot\left[\nabla_{1}^{\prime}-\mathbf{T}_{n_{1} n_{2}}\left(\Omega_{1}^{\prime}, \Omega_{2}\right)\right] \delta \rho_{n_{1} n_{2}}^{(2)}\left(\Omega_{1}^{\prime}, \Omega_{2} ; t\right)+\right. \\
& \left.+\nabla_{2} \cdot \int_{0}^{t} d t^{\prime} \int d \Omega_{2}^{\prime} \mathbf{D}^{\mathrm{eff}}\left(\Omega_{2}, \Omega_{2}^{\prime} ; t-t^{\prime}\right) \cdot\left[\nabla_{2}^{\prime}-\mathbf{T}_{n_{2} n_{1}}\left(\Omega_{2}^{\prime}, \Omega_{1}\right)\right] \delta \rho_{n_{1} n_{2}}^{(2)}\left(\Omega_{1}, \Omega_{2}^{\prime} ; t\right)\right\} \\
& =:\left(\Lambda^{(2)} * \delta \rho^{(2)}\right)_{n_{1} n_{2}}\left(\Omega_{1}, \Omega_{2} ; t\right), \\
& A_{n_{1} n_{2}}^{(2)}\left(\Omega_{1}, \Omega_{2} ; t\right) \approx 0 .
\end{aligned}
$$

The generalized rotational diffusion tensor $\mathbf{D}\left(\Omega, \Omega^{\prime} ; t\right)$ is defined through a constitutive equation relating the 1-rod current density and the (angular) gradient of the 1-rod density

$$
\mathbf{j}_{n_{1}}^{(1)}(\Omega ; t)=-\int_{0}^{t} d t^{\prime} \int d \Omega^{\prime} \mathbf{D}\left(\Omega, \Omega^{\prime} ; t-t^{\prime}\right) \cdot \nabla_{\Omega^{\prime}} \rho_{n_{1}}^{(1)}\left(\Omega^{\prime} ; t\right) .
$$

As the final approximation we impose a self-consistency condition,

$$
\mathbf{D}^{\mathrm{eff}}=\mathbf{D} \text {. }
$$

Eqs. (15 17), (19 22) form a closed set of equations for $\rho^{(1)}, \delta \rho^{(2)}$ and D. Taking their Laplace transform [8] it is straightforward to (formally) eliminate $\rho^{(1)}$ and $\delta \rho^{(2)}$ which results in:

$$
\begin{aligned}
& D_{0}^{-1} \widehat{\mathbf{j}}_{n_{1}}\left(\Omega_{1} ; z\right) \\
& +\sum_{n_{3}} \frac{1}{4 \pi} \int d \Omega_{3} \mathbf{T}_{n_{1} n_{3}}\left(\Omega_{1}, \Omega_{3}\right)\left[\left(z-\widehat{\Lambda}^{(2)}\right)^{-1} *\left(\mathbf{T}_{n_{1} n_{3}} g_{n_{1} n_{3}}^{(2)} \cdot \widehat{\mathbf{j}}_{n_{1}}^{(1)}\right)\right]_{n_{1} n_{3}}\left(\Omega_{1}, \Omega_{3} ; z\right) \\
& =\int d \Omega_{3} \widehat{\mathbf{D}}^{-1}\left(\Omega_{1}, \Omega_{3} ; z\right) \cdot \widehat{\mathbf{j}}_{n_{1}}\left(\Omega_{3} ; z\right)
\end{aligned}
$$

where - denotes the Laplace transformed quantities. From Eq. (23) we find immediately the self-consistent equation for $\widehat{\mathbf{D}}\left(\Omega_{1}, \Omega_{2} ; z\right)$ :

$\S$ Here we use the definition $\widehat{f}(z)=\int_{0}^{\infty} d t f(t) \exp (-z t)$, Re $z>0$ and take $\delta \rho_{n_{1} n_{2}}^{(2)}\left(\Omega_{1}, \Omega_{2} ; t=0\right)=0$ into account. 


$$
\begin{aligned}
& \widehat{\mathbf{D}}^{-1}\left(\Omega_{1}, \Omega_{2} ; z\right)=\mathbf{D}_{0}^{-1} \delta\left(\Omega_{1} \mid \Omega_{2}\right) \\
& +\frac{1}{4 \pi} \sum_{n_{3}} \mathbf{T}_{n_{1} n_{3}}\left(\Omega_{1}, \Omega_{2}\right)\left[\left(z-\widehat{\Lambda}^{(2)}\right)^{-1} *\left(\mathbf{T}_{n_{1} n_{3}}^{t} g_{n_{1} n_{3}}^{(2)}\right)\right]_{n_{1} n_{3}}\left(\Omega_{1}, \Omega_{2} ; z\right)
\end{aligned}
$$

Taking the limit $z \rightarrow 0$ in Eq. (24) and operating with $(1 / 4 \pi) \int d \Omega_{1} \int d \Omega_{2}$ on both sides of the resulting equation yields the following for the diffusion tensor $\mathbf{D}\left(\Omega_{1}, \Omega_{2}\right) \equiv$ $\widehat{\mathbf{D}}\left(\Omega_{1}, \Omega_{2} ; z=0\right)$ :

$$
\left.4 \pi\left\langle\mathbf{D}^{-1}\right\rangle=\mathbf{D}_{0}^{-1}-\sum_{n_{3}}\left\langle\left(\widehat{\Lambda}^{(2) \dagger}\right)^{-1} * \mathbf{T}\right)_{n_{1} n_{3}} \mathbf{T}_{n_{1} n_{3}}^{t}\right\rangle
$$

with

$$
\begin{aligned}
& \left\langle f_{n_{1} n_{2}} h_{n_{1} n_{2}}\right\rangle \\
& =\frac{1}{(4 \pi)^{2}} \int d \Omega_{1} \int d \Omega_{2} g_{n_{1} n_{2}}^{(2)}\left(\Omega_{1}, \Omega_{2}\right) f_{n_{1} n_{2}}^{*}\left(\Omega_{1}, \Omega_{2}\right) h_{n_{1} n_{2}}\left(\Omega_{1}, \Omega_{2}\right),
\end{aligned}
$$

and

$$
\left(\mathbf{D}_{0}\right)^{\alpha \beta} \quad=D_{0} \delta^{\alpha \beta}
$$

$\mathbf{T}^{t}$ is the transpose of $\mathbf{T}$ and for the adjoint 2-rod operator we find from Eq. (19):

$$
\begin{aligned}
& \left(\widehat{\Lambda}^{(2) \dagger} * f\right)_{n_{1} n_{2}}\left(\Omega_{1}, \Omega_{2}\right) \\
& =\left[\nabla_{1}+\mathbf{T}_{n_{1} n_{2}}\left(\Omega_{1}, \Omega_{2}\right)\right] \cdot \int d \Omega_{3} \mathbf{D}\left(\Omega_{3}, \Omega_{1}\right) \cdot \nabla_{3} f_{n_{1} n_{2}}\left(\Omega_{3}, \Omega_{2}\right) \\
& +\left[\nabla_{2}+\mathbf{T}_{n_{2} n_{1}}\left(\Omega_{2}, \Omega_{1}\right)\right] \cdot \int d \Omega_{3} \mathbf{D}\left(\Omega_{3}, \Omega_{2}\right) \cdot \nabla_{3} f_{n_{1} n_{2}}\left(\Omega_{1}, \Omega_{3}\right) .
\end{aligned}
$$

The reader should note that (i) $g_{n_{1} n_{2}}^{(2)}\left(\Omega_{1}, \Omega_{2}\right)$ in Eq. (26) can be skipped, because it is equal to one almost everywhere; of course, it must not be dropped if $\nabla_{1}$ or $\nabla_{2}$ act on it, like in Eq. (18); in that case one can use Eq. (9); (ii) $\left\langle\mathbf{D}^{-1}\right\rangle$ does not depend on $n_{1}$ and $n_{2}$ and (iii) the tensor $\left\langle\left(\left(\widehat{\Lambda}^{(2) \dagger}\right)^{-1} * \mathbf{T}\right)_{n_{1} n_{3}} \mathbf{T}_{n_{1} n_{3}}^{t}\right\rangle$ in Eq. (25) depends only on $\mathbf{R}_{n_{3}}-\mathbf{R}_{n_{1}}$ because of the lattice translational invariance. Accordingly $\sum_{n_{3}}\langle\cdots\rangle$ does not depend on $n_{1}$. Eq. (25) can also be rewritten as follows

$$
4 \pi\left\langle\mathbf{D}^{-1}\right\rangle=\mathbf{D}_{0}^{-1}+\sum_{n_{3}} \int_{0}^{\infty} d t\left\langle\left(e^{\widehat{\Lambda}^{(2) \dagger} t} * \mathbf{T}\right)_{n_{1} n_{3}} \mathbf{T}_{n_{1} n_{3}}^{t}\right\rangle .
$$

Let us discuss Eq. (29). It is a functional equation for the rotational diffusion tensor D, since $\widehat{\Lambda}^{(2) \dagger}$ also involves D. It $l \leq 1$ then $\mathbf{T}_{n_{1} n_{2}}\left(\Omega_{1}, \Omega_{2}\right) \equiv 0$ and therefore Eq. (29) implies that

$$
\mathbf{D}\left(\Omega_{1}, \Omega_{2}\right)=\mathbf{D}_{0} \delta\left(\Omega_{1} \mid \Omega_{2}\right)
$$

as it should be. Increasing $l$ beyond one leads to an "increase" of the friction tensor $\left\langle\mathbf{D}^{-1}\right\rangle$, due to the positive definite, time-dependent torque-torque correlation 
tensor $\left\langle\left(\exp \left(\widehat{\Lambda}^{(2) \dagger} t\right) * \mathbf{T}\right)_{n_{1} n_{3}} \mathbf{T}_{n_{1} n_{3}}^{t}\right\rangle$ which determines the second term in Eq. (29). This "increase" of the "renormalized" friction tensor $\mathbf{D}^{-1}$ implies a decrease of the "renormalized" diffusion tensor D. Since this one enters into the exponent of $\exp \left(\widehat{\Lambda}^{(2) \dagger} t\right)$ the relaxation of the torque-torque correlation will slow down. This in turn leads to an "increase" of the second term of Eq. (29) leading to a further "increase" of $\mathbf{D}^{-1}$ and so on. This feedback mechanism, which is different from but still resembles that of MCT [14, 15, 16], finally may lead to the vanishing of diffusion and therefore to a glass transition.

Although the functional equation possesses a rather clear structure it probably cannot be solved exactly. For this one would have to determine the eigenvalues and eigenfunctions of $\widehat{\Lambda}^{(2) \dagger}$. Due to the singular character of $\mathbf{T}_{n_{1} n_{2}}\left(\Omega_{1}, \Omega_{2}\right)$ (cf. Eq. (8)) on the three-dimensional contact-hypersurface defined by Eq. (11) this does not seem to be feasible. In order to make progress and to get explicit results for $\mathbf{D}$ we will use the following additional approximation:

$$
\left(\mathbf{D}\left(\Omega_{1}, \Omega_{2}\right)\right)^{\alpha \beta} \approx D(l) \delta^{\alpha \beta} \delta\left(\Omega_{1} \mid \Omega_{2}\right) .
$$

Substituting Eq. (31) into Eq. (29) and acting with $\frac{1}{3} \sum_{\alpha, \beta}$ on both sides yields:

$$
D(l)=D_{0}[1-v(l)] \quad .
$$

The l-dependent coupling function $v(l)$ follows from the second term of Eq. (29) by use of Eqs. (28) and (31):

$$
v(l)=\frac{1}{3} \sum_{n_{3}} \int_{0}^{\infty} d t\left\langle\mathbf{T}_{n_{1} n_{3}} \cdot e^{\mathcal{L}^{(2) \dagger} t} \mathbf{T}_{n_{1} n_{3}}\right\rangle
$$

with the adjoint of the 2-rod Smoluchowski operator (cf. Eq. (12)):

$$
\mathcal{L}^{(2) \dagger}=\left[\nabla_{1}+\mathbf{T}_{n_{1} n_{2}}\left(\Omega_{1}, \Omega_{2}\right)\right] \cdot \nabla_{1}+(1 \leftrightarrow 2)
$$

where $D_{0}$ is replaced by one. The calculation of the relevant quantity $v(l)$ and the determination of a critical length $l_{c}$ at which $D(l)$ vanishes will be presented in the next section.

\section{RESULTS}

\subsection{Glass transition singularity}

Using the local approximation Eq. (31), we obtained Eq. (32) which constitutes a rather simple result. Before we calculate $v(l)$ let us discuss $D(l)$ and $v(l)$ on a qualitative level. Since $\mathbf{T}_{n_{1} n_{2}}\left(\Omega_{1}, \Omega_{2}\right) \equiv 0$ for $l \leq 1$ it follows that $v(l)=0$ and therefore $D(l)=D_{0}$ for $l \leq 1$, as it should be. Next, we introduce:

$$
v_{0}\left(l / r_{n}\right)=\frac{1}{3} \int_{0}^{\infty} d t\left\langle\mathbf{T}_{0 \mathrm{n}} \cdot e^{\mathcal{L}^{(2) \dagger} t} \mathbf{T}_{0 \mathrm{n}}\right\rangle
$$


where $r_{n}=\left|\mathbf{R}_{n}\right| / a$. This is related to the torque-torque correlator of one rod at the origin and another one at site $n$. Note that the r.h.s. of Eq. (35) depends on $\left|\mathbf{R}_{n}\right|$, but not on the direction of $\mathbf{R}_{n} . v_{0}(x)$, of course, vanishes for $x \leq 1$, is positive for $x>1$ (see below) and we expect it to converge to a finite limit $v_{0}^{\infty}$ for $x \rightarrow \infty . v(l)$ is completely determined by $v_{0}(x)$ :

$$
v(l)=\sum_{n} v_{0}\left(l / r_{n}\right)
$$

where the sum is restricted to such $n$ for which $r_{n}<l$. This relation can be used to determine the asymptotic behavior of $v(l)$ for $l \rightarrow \infty$. Approximating the sum by an integral which becomes more and more accurate with increasing $r_{n}$ we get:

$$
\begin{aligned}
& v(l) \approx \int_{1 \leq r \leq l} d^{3} r v_{0}(l / r)=4 \pi \int_{1}^{l} d r r^{2} v_{0}(l / r) \\
& =4 \pi\left(\int_{l^{-1}}^{1} d x x^{2} v_{0}\left(\frac{1}{x}\right)\right) l^{3}
\end{aligned}
$$

which gives

$$
v(l) \approx c \cdot l^{3}+O\left(l^{2}\right) \quad, \quad l \rightarrow \infty
$$

with

$$
c=4 \pi \int_{0}^{1} d x x^{2} \quad v_{0}\left(\frac{1}{x}\right)>0 .
$$

That $v(l) \sim l^{3}$ for $l \rightarrow \infty$ is obvious since all rods within a sphere of radius of order $l$ will collide with the central one. Their number, of course, is proportional to $l^{3}$. Because $v(l)=0$ for $l \leq 1$ and $v(l)$ increases linearly with $l^{3}$ for large $l$ there must exist a critical length $l_{c}$ for which $v\left(l_{c}\right)=1$ and therefore $D\left(l_{c}\right)=0$. To determine $l_{c}$ at which a dynamical glass transition takes place we have to calculate $v_{0}(x)$. Although this quantity is much simpler than the second term in Eq. (29), it cannot be determined exactly analytically (see below). Therefore the exact evaluation can only be done numerically. For such a numerically exact calculation we performed a simulation of the Brownian dynamics (defined by $\mathcal{L}^{(2) \dagger}$ ) of an isolated pair of rods with distance $r_{n}$. The timedependent torque-torque correlator resulting from this simulation is shown in Fig. 5 for several values of $l / r_{n}$. For $t \rightarrow 0$ we find a power law divergence $t^{-1 / 2}$ as expected and already proven for the force-force correlator of hard spheres [36, 37]. Since only two rods are considered, the system is ergodic and therefore the torque-correlations relax to zero. Figure 5 shows that the relaxational behavior changes qualitatively around $l / r_{n}=2$ from a fast decay like for free Brownian dynamics to a rather slow relaxation. This crossover at $l / r_{n} \approx 2$ is related to the properties discussed in section 2 . Whether the long time decay of the torque-torque correlation is purely exponential, proportional 
to $t^{-\alpha} \exp (-\lambda t)$ or even a single power law $t^{-\alpha}$ (as it is true for two hard spheres [36]) cannot be decided from our numerical data. Although the log-linear representation in Figure 5 exhibits a bending for large $t$ (which would indicate deviation from a pure exponential) the exponential behavior could appear on a much larger time scale on which the statistical fluctuations of the numerical data prevent the determination of the precise long time decay. The numerical evaluation of the time integral in Eq. (35) leads to $v_{0}^{\text {num }}(x)$ presented in Figure 6. The reader should note the strong increase starting at $x=l / r_{n} \approx 2$.

Now let us turn to the analytical calculation of $v_{0}(x)$. For this we rewrite $v_{0}(x)$ as

$$
v_{0}(x)=\frac{1}{3}\left\langle\mathbf{T}_{0 \mathrm{n}} \cdot\left(-\mathcal{L}^{(2) \dagger}\right)^{-1} \mathbf{T}_{0 \mathrm{n}}\right\rangle .
$$

which is (up to the factor 1/3) the Laplace transformed torque-torque correlator at $z=0$.

Analogous to refs. [26, 27] we introduce a vector function $\mathbf{f}_{n_{1} n_{2}}\left(\Omega_{1}, \Omega_{2}\right)$ such that:

$$
\mathcal{L}^{(2) \dagger} \mathbf{f}_{n_{1} n_{2}}\left(\Omega_{1}, \Omega_{2}\right)=\mathbf{T}_{n_{1} n_{2}}\left(\Omega_{1}, \Omega_{2}\right) .
$$

Eq. (40) can be decomposed into a regular part

$$
\left[\nabla_{1}^{2}+\nabla_{2}^{2}\right] \mathbf{f}_{n_{1} n_{2}}\left(\Omega_{1}, \Omega_{2}\right)=0
$$

and a singular one:

$$
\left[s_{12}\left(\mathbf{u}_{1} \times \widehat{\mathbf{r}}_{12}^{\perp}\right) \cdot \nabla_{1}+s_{21}\left(\mathbf{u}_{2} \times \widehat{\mathbf{r}}_{21}^{\perp}\right) \cdot \nabla_{2}\right] \mathbf{f}_{n_{1} n_{2}}\left(\Omega_{1}, \Omega_{2}\right)=s_{12}\left(\mathbf{u}_{1} \times \widehat{\mathbf{r}}_{12}^{\perp}\right) .
$$

Eq. (42) is to be satisfied for $\Omega_{1}, \Omega_{2}$ located on the three-dimensional contact hypersurface. Accordingly, Eqs. (41-42) describe a boundary value problem with boundary value on the three-dimensional hypersurface embedded in the four-dimensional space built by the surface of two unit spheres. This is a difficult mathematical problem which seems to resist a rigorous solution. If it could be solved then the calculation of $v_{0}(x)$ is reduced to the calculation of an integral over $\Omega_{1}$ and $\Omega_{2}$ :

$$
v_{0}(x)=-\frac{1}{3}\left\langle\mathbf{T}_{0 \mathrm{n}} \cdot \mathbf{f}_{0 \mathrm{n}}\right\rangle .
$$

Therefore, we resort to an alternative. It is easy to prove (by the use of Eq. (9)) that Eq. (40) is the variational equation of the functional:

$$
\mathcal{F}\left[\mathbf{f}_{n_{1} n_{2}}\left(\Omega_{1}, \Omega_{2}\right)\right]=\frac{1}{3}\left[\left\langle\mathbf{f}_{n_{1} n_{2}} \cdot \mathcal{L}^{(2) \dagger} \mathbf{f}_{n_{1} n_{2}}\right\rangle-2\left\langle\mathbf{T}_{n_{1} n_{2}} \cdot \mathbf{f}_{n_{1} n_{2}}\right\rangle\right] .
$$

For a similar discussion see ref. [38. It can be easily shown by partial integration that:

$$
\begin{aligned}
& \left\langle\mathbf{f}_{n_{1} n_{2}} \cdot \mathcal{L}^{(2) \dagger} \mathbf{f}_{n_{1} n_{2}}\right\rangle= \\
& -\frac{1}{(4 \pi)^{2}} \int d \Omega_{1} \int d \Omega_{2} g_{n_{1} n_{2}}^{(2)}\left(\Omega_{1}, \Omega_{2}\right) \sum_{\alpha}\left[\left(\nabla_{1} f_{n_{1} n_{2}}^{\alpha}\left(\Omega_{1}, \Omega_{2}\right)\right)^{2}\right. \\
& \left.+\left(\nabla_{2} f_{n_{1} n_{2}}^{\alpha}\left(\Omega_{1}, \Omega_{2}\right)\right)^{2}\right]
\end{aligned}
$$


which is negative for all $\mathbf{f}_{n_{1} n_{2}} \neq$ const, i.e. $\mathcal{L}^{(2) \dagger}$ is a negative operator. Therefore one can follow ref. [38] and prove that the solution $\mathbf{f}_{n_{1} n_{2}}^{\text {sol }}\left(\Omega_{1}, \Omega_{2}\right)$ of Eq. (40) is the non-degenerate maximum of $\mathcal{F}$. From Eqs. (40), (43) and (44) we then find that

$$
v_{0}(x)=\mathcal{F}\left[\mathbf{f}_{n_{1} n_{2}}^{\mathrm{sol}}\left(\Omega_{1}, \Omega_{2}\right)\right],
$$

i.e. the maximum value of $\mathcal{F}$ is just $v_{0}(x)$. Since the maximum is non-degenerate the following inequality is true for any trial function $\mathbf{f}_{n_{1} n_{2}}^{\mathrm{var}}\left(\Omega_{1}, \Omega_{2}\right)$

$$
v_{0}^{\operatorname{var}}(x) \equiv \mathcal{F}\left[\mathbf{f}_{n_{1} n_{2}}^{\operatorname{var}}\left(\Omega_{1}, \Omega_{2}\right)\right] \leq v_{0}(x) .
$$

Choosing an appropriate trial function offers the possibility to determine a lower bound for $v_{0}(x)$ and therefore an upper bound for the critical length $l_{c}$.

The boundary value problem described above is similar to those arising from electrodynamics or hydrodynamics. Its singular part implies that $\mathbf{f}_{n_{1} n_{2}}\left(\Omega_{1}, \Omega_{2}\right)$ is discontinuous at the contact surface. This is similar to the behavior of the electric field at a dipolar layer. Without restricting generality, we can choose $\mathbf{R}_{n_{2}}-\mathbf{R}_{n_{1}}$ along the $z$-axis. Then the relevant coordinate is $\phi=\phi_{2}-\phi_{1}$ and contacts can occur at $\phi=0^{ \pm}$or $\phi=\pi^{ \pm}$(see discussion in section 2), i.e. $\mathbf{f}_{n_{1} n_{2}}\left(\Omega_{n_{1}}, \Omega_{n_{2}}\right) \equiv \mathbf{f}_{n_{1} n_{2}}\left(\theta_{1}, \theta_{2}, \phi_{1}, \phi\right)$ must be discontinuous at $\phi=0$ and $\phi=\pi$. Next, Eq. (43) makes it obvious that $\left|\mathbf{T}_{n_{1} n_{2}} \cdot \mathbf{f}_{n_{1} n_{2}}\right|$ should be made as large as possible. This can be done by choosing $\mathbf{f}_{n_{1} n_{2}} \sim \mathbf{T}_{n_{1} n_{2}} \sim \mathbf{u}_{1} \times \widehat{\mathbf{r}}_{12}^{\perp}=\left(\cos \theta_{1} \cos \phi_{1}, \cos \theta_{1} \sin \phi_{1},-\sin \theta_{1}\right)$. Finally, the calculation of $\mathcal{F}$ from Eq. (44) requires the calculation of the r.h.s. of Eq. (45). Since $\nabla_{i} f_{n_{1} n_{2}}^{\alpha}\left(\Omega_{1}, \Omega_{2}\right)$ involves $1 / \sin \theta_{i}$ for $x$ - and $y$ - component of the gradient, the integrals in Eq. (45) will not exist unless $f_{n_{1} n_{2}}^{\alpha} \sim\left(\sin \theta_{1}\right)^{\nu_{1}}\left(\sin \theta_{2}\right)^{\nu_{2}}$ with $\nu_{1} \geq 1$ and $\nu_{2} \geq 1$. These considerations suggest to use the following trial function:

$$
\begin{aligned}
\mathbf{f}_{n_{1} n_{2}}^{\mathrm{var}}\left(\Omega_{1}, \Omega_{2}\right) \equiv & \lambda \mathbf{h}_{n_{1} n_{2}}^{\mathrm{var}}\left(\Omega_{1}, \Omega_{2}\right)=\lambda \sin \theta_{1} \sin \theta_{2}\left(\begin{array}{c}
\cos \theta_{1} \cos \phi_{1} \\
\cos \theta_{1} \sin \phi_{1} \\
-\sin \theta_{1}
\end{array}\right) \\
& \times \begin{cases}\frac{\pi}{2}-\left(\phi_{2}-\phi_{1}\right), & 0<\phi_{2}-\phi_{1}<\pi \\
\frac{3 \pi}{2}-\left(\phi_{2}-\phi_{1}\right), & \pi<\phi_{2}-\phi_{1}<2 \pi\end{cases}
\end{aligned}
$$

with $\lambda$ being a variational parameter. For the $\left(\phi_{2}-\phi_{1}\right)$-dependence we have made the simplest choice taking a linear variation with discontinuities at 0 and $\pi$. Note also that $\mathbf{f}_{n_{1} n_{2}}^{\mathrm{var}}\left(\Omega_{1}, \Omega_{2}\right)$ does not depend on either $\mathbf{R}_{n_{2}}-\mathbf{R}_{n_{1}}$ or $x=l / r_{n}$, which will be not true for the true, exact solution $\mathbf{f}_{n_{1} n_{2}}^{\text {sol }}\left(\Omega_{1}, \Omega_{2}\right)$. Substituting $\mathbf{f}_{n_{1} n_{2}}^{\text {var }}\left(\Omega_{1}, \Omega_{2}\right)$ into Eq. (44), where the first term is evaluated by Eq. (45), one obtains:

$$
\mathcal{F}\left[\mathbf{f}_{n_{1} n_{2}}^{\mathrm{var}}\left(\Omega_{1}, \Omega_{2}\right)\right]=F(\lambda)=\lambda^{2} I_{2}-2 \lambda I_{1}(x)
$$

with

$$
I_{1}(x)=\frac{1}{3} \frac{1}{(4 \pi)^{2}} \int d \Omega_{1} \int d \Omega_{2} \mathbf{h}_{n_{1} n_{2}}^{\mathrm{var}}\left(\Omega_{1}, \Omega_{2}\right) \cdot \mathbf{T}_{n_{1} n_{2}}\left(\Omega_{1}, \Omega_{2}\right)
$$




$$
\begin{aligned}
& I_{2}=-\frac{1}{3} \frac{1}{(4 \pi)^{2}} \\
& \times \int d \Omega_{1} \int d \Omega_{2} \sum_{\alpha}\left[\left(\nabla_{1} h_{n_{1} n_{2}}^{\mathrm{var}, \alpha}\left(\Omega_{1}, \Omega_{2}\right)\right)^{2}+\left(\nabla_{2} h_{n_{1} n_{2}}^{\mathrm{var}, \alpha}\left(\Omega_{1}, \Omega_{2}\right)\right)^{2}\right] .
\end{aligned}
$$

Note that $I_{2}$ does not depend on $x . F(\lambda)$ is easily maximized. As a result one obtains:

$$
v_{0}^{\operatorname{var}}(x)=F\left(\lambda_{\max }\right)=\frac{\left(I_{1}(x)\right)^{2}}{\left(-I_{2}\right)} .
$$

$I_{2}$ can be calculated analytically:

$$
I_{2}=-\frac{2}{81}\left(27+2 \pi^{2}\right) \cong-1.154
$$

whereas $I_{1}(x)$ must be calculated by numerical integration, due to the nontrivial contact hypersurface. The result $v_{0}^{\mathrm{var}}(x)$ following from this approach is shown in Fig. 6. It is interesting that $v_{0}^{\mathrm{var}}(x)$ reproduces the crossover behavior found for $v_{0}^{\text {num }}(x)$ close to $x=2$ from $v_{0}^{\operatorname{var}}(x) \approx 0$ for $x<2$ to $v_{0}^{\operatorname{var}}(x)>0$ for $x>2$. Comparison of $v_{0}^{\text {num }}(x)$ with $v_{0}^{\operatorname{var}}(x)$ confirms that $v_{0}^{\operatorname{var}}(x)$ is a lower bound for $v_{0}^{\text {num }}(x)$, as it should be. Introducing $v_{0}(x)$ from both approaches into Eq. (36) leads to $v(l)$ represented in Fig. 7 for an sc-lattice. From this figure we get:

$$
l_{c}^{\text {num }} \cong 3.45 \quad, \quad l_{c}^{\mathrm{var}} \cong 5.68 \quad: \quad \text { sc lattice } .
$$

$v(l)$ can be calculated for any periodic lattice. For the other cubic lattices we obtained

$$
l_{c}^{\text {num }} \cong\left\{\begin{array}{l}
2.78 \\
2.20
\end{array} \quad, \quad l_{c}^{\mathrm{var}} \cong \begin{cases}4.84 & \text { bcc lattice } \\
3.96 & \text { fcc lattice }\end{cases}\right.
$$

Since $v^{\mathrm{var}}(l) \leq v^{\text {num }}(l) \cong v(l)$, it follows that $l_{c}^{\mathrm{var}} \geq l_{c}^{\text {num }} \cong l_{c}$. The critical length decreases when going from sc to bcc to fcc lattice. This decrease is related to (i) the increase of the coordination number $z$ from 6 to 8 to 12 and (ii) a decrease of the nearest neighbor distances $r_{1}$ from 1 to $\sqrt{3} / 2$ to $\sqrt{2} / 2$ (in units of the lattice constant $a$ ). Since an increase of $z$ and a decrease of $r_{1}$ results in an increase of the steric hindrance, $l_{c}$ must decrease. As the increase of steric hindrance is equivalent to an increase of collisions, $i$. $e$. of contacts, our result has some similarity to that found in ref. [39]. These authors have used a probabilistic approach in order to derive a criterion for the mechanical stability of an off-lattice system of infinitely thin hard rods in its randomly closed-packed glassy state. It has been found that a mechanically stable amorphous phase occurs if the average contact point per rod becomes 5 . However, such an arrangement of hard rods with $d=0$ is dynamically unstable, as discussed in the Introduction. In the simulation performed in ref. [29] it was found $l_{c}^{\text {sim }} \cong 2.7$ for an fcc lattice. On the other hand ref. [30] found $l_{c}^{\text {sim }} \cong 4.5$ for an sc lattice. Note that in ref. [30] the rods were fixed with one of their endpoints, and not with their centers. Our result for $l_{c}^{\text {num }}$ and $l_{c}^{\text {var }}$ agree 
satisfactorily to the simulational result of ref. [29]. The difference between our result and that of ref. [30] may be due to the different way of fixing rods on the lattice that was mentioned above.

The authors of ref. 29] have also studied the $l$-dependence of the rotational diffusion constant $D(l)$ defined by:

$$
D(l)=\lim _{t \rightarrow \infty}\left(-\frac{1}{2 t} \ln \left[\frac{1}{N} \sum_{n=1}^{N}\left\langle\mathbf{u}_{n}(t) \cdot \mathbf{u}_{n}(0)\right\rangle\right]\right) \quad .
$$

The variation of $D$ over about two orders in magnitude follows a power law $D(l) \sim$ $\left(l_{c}-l\right)^{\gamma^{M D}}$ with $\gamma^{M D} \cong 4.2$. Since $v(l)$ is analytical for $l>1$ it follows that $1-v(l) \sim l_{c}-l$ and therefore $D(l) \sim\left(l_{c}-l\right)$. Hence our analytical theory yields $\gamma=1$ which differs significantly from $\gamma^{M D}$. This deviation may have two reasons. First, our result $\gamma=1$ is mean-field-like (see, e.g., ref. [22]). Note that in ref. [22] going beyond mean field approximation it has been found $\gamma \cong 7 / 6$ [22] which is still close to one. Second, $\gamma^{M D} \cong 4.2$ is unusually high. Simulations and experiments of supercooled liquids usually yield $\gamma \approx 2$ consistent with most MCT-analyses [14, 15, 16, 18, 19, 20].

\subsection{Nonergodicity parameter and dynamics close to $l_{c}$}

The analysis in subsection 4.1 has proven that our theory predicts a dynamical glass transition at a critical length $l_{c}$. There are two important questions remaining. First, does the nonergodicity parameter change at $l_{c}$ in a continuous (type-A transition [14]) or discontinuous (type-B transition [14]) way? Second, what is the time- or frequencydependence of the 1-rod density $\rho_{n}^{(1)}$ close to $l_{c}$ ? The present subsection will give answers to both questions.

Let us expand $\widehat{\rho}_{n}^{(1)}(\Omega ; z)$ and $\widehat{\mathbf{j}}_{n}^{(1)}(\Omega ; z)$ into spherical harmonics:

$$
\begin{gathered}
\widehat{\rho}_{n}^{(1)}(\Omega ; z)=\sum_{\lambda} \widehat{\rho}_{n, \lambda}^{(1)}(z) Y_{\lambda}(\Omega) \\
\widehat{\mathbf{j}}_{n}^{(1)}(\Omega ; z)=\sum_{\lambda} \widehat{\mathbf{j}}_{n, \lambda}^{(1)}(z) Y_{\lambda}(\Omega) .
\end{gathered}
$$

where $\lambda=(j, m), j=0,1,2, \cdots ;-j \leq m \leq j$. Furthermore, let us extend approximation (31) to finite $z$ :

$$
\left(\widehat{\mathbf{D}}\left(\Omega_{1}, \Omega_{2} ; z\right)\right)^{\alpha \beta} \approx \widehat{D}(z) \delta^{\alpha \beta} \delta\left(\Omega_{1} \mid \Omega_{2}\right)
$$

It is easy to prove then that the Laplace transform of Eqs. (15) and (21) leads to

$$
\widehat{\phi}_{\lambda}(z) \equiv \widehat{\rho}_{\lambda}^{(1)}(z) / \rho_{\lambda}^{(1)}(t=0)=1 /[z+j(j+1) \widehat{D}(z)]
$$

where we skipped the index $n$.

The nonergodicity parameter $f_{\lambda}$ is defined as follows:

$$
f_{\lambda}=\lim _{z \rightarrow 0} z \widehat{\phi}_{\lambda}(z) .
$$


Taking into account approximation Eq. (57) we get from Eq. (24) after operating with $(1 / 4 \pi) \int d \Omega_{1} \int d \Omega_{2}$ on both sides the self-consistency equation for $\widehat{D}(z)$ :

$$
\frac{1}{\widehat{D}(z)}=\frac{1}{D_{0}}+\frac{1}{\widehat{D}(z)} \widehat{v}(\zeta ; l), \quad \zeta=z / \widehat{D}(z),
$$

with

$$
\widehat{v}(\zeta ; l)=\frac{1}{3} \sum_{n}\left\langle\mathbf{T}_{0 \mathrm{n}} \cdot\left[\zeta-\mathcal{L}^{(2) \dagger}\right]^{-1} \mathbf{T}_{0 \mathrm{n}}\right\rangle .
$$

Inverse Laplace transform of Eq. (61) yields

$$
v(t ; l)=\frac{1}{3} \sum_{n}\left\langle\mathbf{T}_{0 \mathrm{n}} \cdot e^{\mathcal{L}^{(2) \dagger} t} \mathbf{T}_{0 \mathrm{n}}\right\rangle .
$$

Note that $v(t ; l)$ is the time-dependent analogue of $v(l)$ defined in Eq. (33).

Eq. (60) has a similar mathematical structure as the corresponding equation for a Lorentz gas obtained from a mode coupling approximation 40, 41]. Assuming that $F(t)$ does not decay slower than $t^{-5 / 2}$ for $t \rightarrow \infty$ [42] it follows from the Tauberian theorems 43 that

$$
\widehat{v}(\zeta ; l)=\widehat{v}(0 ; l)+\widehat{v}^{\prime}(0 ; l) \zeta+O\left(\zeta^{3 / 2}\right)
$$

where

$$
\begin{aligned}
& \widehat{v}(0 ; l) \equiv v(l) \\
& \widehat{v}^{\prime}(0 ; l)=-\frac{1}{3} \sum_{n}\left\langle\mathbf{T}_{0 \mathrm{n}} \cdot\left(\mathcal{L}^{(2) \dagger}\right)^{-2} \mathbf{T}_{0 \mathrm{n}}\right\rangle:=-a(l)<0 .
\end{aligned}
$$

Substituting Eq. (6364) into (60) and neglecting $O\left(\zeta^{3 / 2}\right)$ we get a quadratic equation for $z / \widehat{D}(z)$, the physical solution of which is

$$
z / \widehat{D}(z)=\frac{1}{2}\left[\epsilon(l)+\sqrt{\epsilon^{2}(l)+z \cdot t_{0}}\right]
$$

with

$$
\epsilon(l)=\frac{v(l)-1}{a\left(l_{c}\right)}=\left\{\begin{array}{lll}
<0, & l<l_{c} & \text { (ergodic phase) } \\
\geq 0, & l \geq l_{c} & \text { (glass phase) }
\end{array}\right.
$$

and $t_{0}=4 /\left(a\left(l_{c}\right) D_{0}\right)$ being a microscopic time scale. Note that Eq. (65) is identical to the corresponding equation obtained for the so-called $F_{1}$-model [14. Accordingly, all results for the $F_{1}$-model [14] hold here as well. This implies (i) that the glass transition is of type A, i.e. the nonergodicity parameters (obtained from Eqs. (58) and (59))

$$
f_{\lambda}(l)= \begin{cases}0 & , \quad l=\leq l_{c} \\ \frac{\epsilon(l)}{j(j+1)} \cong \frac{v^{\prime}\left(l_{c}\right)}{a\left(l_{c}\right) j(j+1)}\left(l-l_{c}\right), \quad l \rightarrow l_{c}^{+}\end{cases}
$$

vary continuously at $l_{c}$, (ii) at the critical point $l=l_{c}$ and for $z \rightarrow 0, t \rightarrow \infty$

$$
\widehat{\phi}_{\lambda}(z) \cong \frac{1}{2}\left(z t_{0}\right)^{1 / 2}, \quad \phi_{\lambda}(t) \cong \frac{1}{\sqrt{4 \pi}}\left(t / t_{0}\right)^{-1 / 2}
$$

and (iii) for $\left|l-l_{c}\right| \ll 1$ and for $z \rightarrow 0, t \rightarrow \infty$

$$
\widehat{\phi}_{\lambda}(z, \epsilon)=\left(|\epsilon| / \omega_{\epsilon}\right) \widehat{\phi}_{\lambda}\left(z / \omega_{\epsilon}\right), \quad \phi_{\lambda}(t, \epsilon)=|\epsilon| \phi_{\lambda}\left(t / t_{\epsilon}\right)
$$


with $\omega_{\epsilon}=t_{\epsilon}^{-1}=\epsilon^{2} / t_{0}$. In other words, the $(z, \epsilon)$ - and $(t, \epsilon)$-dependence follows from the master function $\widehat{\phi}_{\lambda}$ and $\phi_{\lambda}$ (Eq. (68)), respectively, by use of the scaled frequency $z / \omega_{\epsilon}$ and time $t / t_{\epsilon}$. The time scale $t_{\epsilon}$ diverges as $\epsilon^{-2}$ when approaching the glass transition independent on the sign of $\epsilon$.

\section{SUMMARY AND CONCLUSIONS}

Our main motivation has been the microscopic description of the glass transition for systems with trivial statics, i.e. systems which do not undergo an equilibrium phase transition to an ordered phase and do not have static correlations. For such systems, present microscopic theories like MCT and replica theory for structural glasses predict neither a dynamical nor a static glass transition. As a model we have chosen $N$ infinitely thin hard rods with length $L$ fixed with their centers on a periodic lattice with lattice constant $a$. The only relevant physical parameter is the dimensionless length $l=L / a$. Simulations for an fcc [29] and an sc lattice [30] strongly suggested that a dynamical glass transition occurs at a critical length $l_{c}^{\text {sim }} \cong 2.7$ [29] and $l_{c}^{\text {sim }} \cong 4.5$ [30. Note that the rods in ref. [30] were fixed at the lattice sites with one of their end points.

To describe the dynamics of our model we have used the generalized $N$-rod Smoluchowski equation from which we derived a hierarchy of coupled equations for $\delta \rho_{n_{1} \cdots n_{k}}^{(k)}\left(\Omega_{1}, \cdots, \Omega_{k}\right)$, the fluctuations of the reduced $k$-rod densities. Truncating at the second level and approximating the influence of surrounding rods on a pair of rods by the introduction of an effective diffusion tensor $\mathbf{D}$ we finally obtained a self-consistency equation for $\mathbf{D}$ (Eq. (25)). This equation describes a feedback mechanism (as MCT also does) which leads to a slowing down of the dynamics and ultimately to an orientational glass transition, similar to that occuring in plastic crystals [5, 6, 7, 8, 11, 12, 13]. The model studied in the present paper may be applied to real plastic crystals as has been shown in ref. [8].

One of the essential features of present MCT for the glass transition is the cage effect [14. For low temperature or high density an extended particle, e.g. a hard sphere, is captured in a cage. The occurrence of this cage is accompanied by the growth of static correlations. Particularly, the main peak of the static structure factor grows and this leads to an increase of the static vertices entering the MCT-equations. If the vertices reach a critical strength the system will undergo a dynamical glass transition, provided ordering is prevented. In this sense the cage effect entering into MCT through the static correlations is of static nature. The model studied here does not have any static correlations, yet there is a cage effect. If the rods are long enough the tagged rod's motion can be strongly restricted to an "orientational" cage (cf. Figure 2). This cage, however, is of pure dynamical nature and leads to a glass transition as well.

Comparing the results for the fcc lattice obtained from our theoretical framework with the additional approximation Eq. (31) with those from simulations [29, 30] one can say that both values $l_{c}^{\text {num }} \cong 2.20$ and $l_{c}^{\mathrm{var}} \cong 3.96$ are in a reasonable range of $l_{c}^{\mathrm{sim}} \cong 2.7$ [29]. Taking into account that our approach involves uncontrollable approximations 
(Eqs. (19) and (20)) this is a satisfactory result. The tendency of $l_{c}$ to decrease when going from the sc lattice to the bcc to the fcc lattice is consistent with our expectation that $l_{c}$ decreases with increase of the coordination number and decrease of the nearest neighbor distances. Of course, second-, third-, etc. nearest neighbors play also a role, but with minor influence. Less satisfactory is the exponent, $\gamma$ of the power law, $D(l) \sim\left(l_{c}-l\right)^{\gamma}$, which is $\gamma=1$, in contrast to $\gamma^{\mathrm{sim}} \cong 4.2$ [30]. Even if the simulated result turns out too high, the type-A transition result, $\gamma=1$, cannot be the correct value. This requires an improvement of our microscopic approach. It is not obvious whether the approach presented in ref. [22] which is based on rather qualitative arguments can serve as a guide.

It seems that the "dynamical cage effect" leads to a discontinuous variation of the nonergodicity parameter at $l_{c}$ and is accompanied close to $l_{c}$ by a two-step relaxation process, as can be seen from the orientational correlator studied in ref. [29]. Such a two-step relaxation was first predicted by MCT [1, 14, 15, 16] and within this theory it is related to the cage effect. The present theoretical approach predicts a continuous transition for the glass order parameters $f_{\lambda}$. Therefore it does not yield a two-step relaxation. This fact demands for an improvement of our theory, based on the hierarchy of equations for the $k$-rod densities. But on the other hand it is, of course, a big challenge to extend the present MCT such that it includes the "dynamical cage effect" described above and that a discontinuous glass transition occurs. If this turns out to be possible, it seems that the vertices must be generalized. Besides static correlations, i.e. correlators at time $t=0$, they also must contain dynamical ones. These dynamical correlations may enter through a time-dependent force-force correlator $\int_{0}^{\infty} d t\langle\mathbf{F}(t) \cdot \mathbf{F}(0)\rangle$, i.e. as a Laplace transformed correlator at frequency $z=0$ (as found in the present approach). For systems with vanishing static correlations, like for our model, the vertices would be of purely dynamical nature. Now, increasing $d$ to finite values will generate static correlations. For small thickness the "dynamical cage" will be still dominant, but at a crossover value $d_{\text {c.o. }}$ the static cage effect would become comparable with the dynamical one and for $d>d_{\text {c.o. }}$ it would be the dominant one.

Let us come back to the choice of Brownian vs. Newtonian dynamics. We have found that the glass transition is driven by the increase of the Laplace transform at $z=0$ of the time dependent torque-torque correlator of an isolated pair of rods. This correlator is be different for Brownian and Newtonian dynamics. Therefore the critical length $l_{c}$ will be not the same for both dynamics. At present, it is not clear whether this is only a small effect. This is different from what has been found for liquids with nontrivial static correlations [33, 34]. However, the linear dependence of the nonergodicity parameters and of the diffusion constant on $\epsilon$, the quadratic dependence of the frequency scale $\omega_{\epsilon}$ on $\epsilon$ and the $t^{-1 / 2}$ dependence of the correlator at the critical length $l_{c}$ should be independent of the type of dynamics.

It would be interesting to re-investigate the lattice model with infinitely thin hard rods by computer simulations. With present computers it should be possible to cover 
a larger time range and to study the dynamics close to $l_{c}$ in greater detail. This would allow to check whether the two scaling laws and other predictions of MCT are consistent with simulations. If the outcome of such a test proves consistency it would encourage theoretical efforts to extend the microscopic theory within the framework of MCT.

Besides rods on a lattice one could also investigate liquid systems of "particles" built by crossing infinitely thin hard rods. If the number of "legs" (rods) of a "particle" becomes large they may exhibit quite similar dynamical behavior to hard spheres, although there are no static correlations. The time scale on which these "particles" realize that they are not hard spheres could be much larger than a typical time scale for structural relaxation.

To conclude, we have discussed a purely dynamical mechanism which also drives a glass transition and which up to now has received almost no attention. Its further investigation by computer simulations and analytical work seems to us a challenging task for the future.

\section{ACKNOWLEDGMENT}

We gratefully acknowledge a very fruitful and stimulating discussion with W. Götze during the " 3 rd Workshop on Non-Equilibrium Phenomena in Supercooled Fluids, Glasses, and Amorphous Materials" in Pisa and his comments on the present manuscript. Helpful comments by M. Fuchs and the preparation of most of the figures by D. Garanin and M. Ricker are gratefully acknowledged as well. G. S. acknowledges support from the National Science Foundation through grant No. CHE 0111152 and the Alexander von Humboldt Foundation. 
[1] Bengtzelius U, Götze W and Sjölander A 1984 J. Phys. C17 5915

[2] Das SP and Mazenko GF 1986 Phys. Rev. A34 2265

[3] Mézard M and Parisi G 1996 J. Phys. A: Math. Gen 29 6515; 1999 Phys. Rev. Lett. 82747

[4] Singh Y, Stoessel JP and Wolynes PG 1985 Phys. Rev. Lett. 541059

[5] Suga H and Seki S 1974 J. Non-cryst. Solids 16171

[6] Srinivasan A, Bermejo FJ, de Andrés A, Dawidowski J, Zúñiga J and Criado A 1996 Phys. Rev. B53 8172

[7] Ramos MA, Vieira S, Bermejo FJ, Dawidowski J, Fischer HE, Schober H, González MA, Loong CK and Price DL 1997 Phys. Rev. Lett. 7882

[8] Jiménez-Ruiz M, Criado A, Bermejo FJ, Cuello GJ, Trouw FR, Fernández-Perea R, Löwen H, Cabrillo C and Fischer HE 1999 Phys. Rev. Lett. 832757

[9] Michel KH 1986 Phys. Rev. Lett. 57 2188; 1988 Z. Phys. B71 369

[10] Bostoen C and Michel KH 1991 Phys. Rev. B43 4415

[11] Criado A, Jiménez-Ruiz M, Cabrillo C, Bermejo FJ, Fernández-Perea R, Fischer HE and Trouw FR 2000 Phys. Rev. B61 12082

[12] Affouard F, Cochin E, Decressain R and Descamps M 2001 Europhys. Lett. 53611

[13] Affouard F and Descamps M 2001 Phys. Rev. Lett. 87035501

[14] Götze W 1991 Liquids, Freezing and the Glass Transition eds J-P Hansen, D Levesque and J Zinn-Justin (Amsterdam: North-Holland) p 287

[15] Schilling R 1994 Disorder Effects on Relaxational Processes eds R Richert and A Blumen (Berlin: Springer-Verlag) p 193

[16] Cummins HZ 1999 J. Phys.: Condens. Matter 11 A95

[17] Hansen J-P and McDonald IR 1986 Theory of Simple Liquids (London: Academic)

[18] Götze W and Sjögren L 1992 Rep. Prog. Phys. 55241

[19] Götze W 1999 J. Phys.: Condens. Matter 11 A1

[20] Kob W 1997 Experimental and Theoretical Approaches to Supercooled Liquids: Advances and Novel Applications eds J Fourkas, D Kivelson, U Mohanty and K Nelson (Washingtom: ACS Books) p 28

[21] Kob W 1999 J. Phys.: Condens. Matter 11 R85

[22] Edwards SF and Vilgis Th 1986 Physica Scripta T 137

[23] Bergenholtz J and Fuchs M 1999 Phys. Rev. E59 5706

[24] Bergenholtz J and Fuchs M 1999 J. Phys.: Condens. Matter 1110171

[25] Bergenholtz J, Fuchs M and Voigtmann Th 2000 J. Phys.: Condens. Matter 126575

[26] Szamel G 1993 Phys. Rev. Lett. 703744

[27] Szamel G and Schweizer K 1994 J. Chem. Phys. 1003127

[28] Rubinstein M and Obukhov SP unpublished

[29] Renner C, Löwen H and Barrat JL 1995 Phys. Rev. E52 5091

[30] Obukhov SP, Kobsev D, Perchak D and Rubinstein M 1997 J. Phys. I France 7563

[31] Schilling R and Szamel G, Europhys. Lett., in print.

[32] Cichocki B 1987 Z. Phys. B66 537

[33] Szamel G and Löwen H 1991 Phys. Rev. A44 8215

[34] Gleim T, Kob W and Binder K 1998 Phys. Rev. Lett. 814404

[35] Cichocki B 1988 Physica 148A 165

[36] Ackerson BJ and Fleischman L 1982 J. Chem. Phys. 762675

[37] Jones RB 1984 J. Phys. A17 2305

[38] Dhont JKG, van Bruggen PB and Briels WJ 1999 Macromolecules 323809

[39] Philipse AP and Verberkmoes A 1997 Physica A235 186

[40] Götze W, Leutheusser E and Yip S 1981 Phys. Rev. A23 2634; A24, 1008

[41] Götze W, Leutheusser E and Yip S 1982 Phys. Rev. A25 533

[42] Our numerical result (Fig. 5) is consistent with this assumption. It is possible that the decay is exponential due to the compact configuration space of two needles in contrast to two hard 
spheres.

[43] Feller W 1971 An Introduction to Probability Theory and its Applications John Wiley \& Sons, p. $442 \mathrm{ff}$. 
Figure 1. Unit cell of the sc-lattice with lattice constant $a$ with hard rods of length L.

Figure 2. Illustration of the blocking of $\operatorname{rod} 1$ by rods $2-5$ within shaded region for $l>2$.

Figure 3. Illustration of possible collisions of rod $i$ and $j$ for fixed rod $i$. For better visualization a small but finite thickness has been used. (a) Single collision for $l<2$ at $\phi_{j}=\phi_{i}^{ \pm}$. The rod $j^{\prime}$ (dashed) turned around z-axis by $\pi$ does not lead to a second collision if $l<2$. (b) Single collision for $l<2$ at $\phi_{j}=\phi_{i}^{ \pm}+\pi$. Again a rotation by $\pi$ (not shown) does not yield a second collision (c) Two collisions for $l>2 ; \theta_{i}$ and $\theta_{j}$ are chosen such that two collisions occur at $\phi_{j}=\phi_{i}^{ \pm}$and $\phi_{j}=\phi_{i}^{ \pm}+\pi$.

Figure 4. Illustration of geometrical quantities defined in the text for two rods $i$ and $j$.

Figure 5. Time-dependence of the torque-torque correlator of an isolated pair of rods for different $l / r_{n}$. Dotted line: $l / r_{n}=1.8$; dash-dotted line: $l / r_{n}=2$; dashed line: $l / r_{n}=4 ;$ solid line: $l / r_{n}=6$

Figure 6. $v_{0}(x)$ from the numerically exact (squares) and the variational calculation (solid line).

Figure 7. $v(l)$ for a sc-lattice from the numerically exact (squares) and the variational calculation (solid line). $l_{c}$ denotes the critical length for which $v\left(l_{c}\right)=1$. 


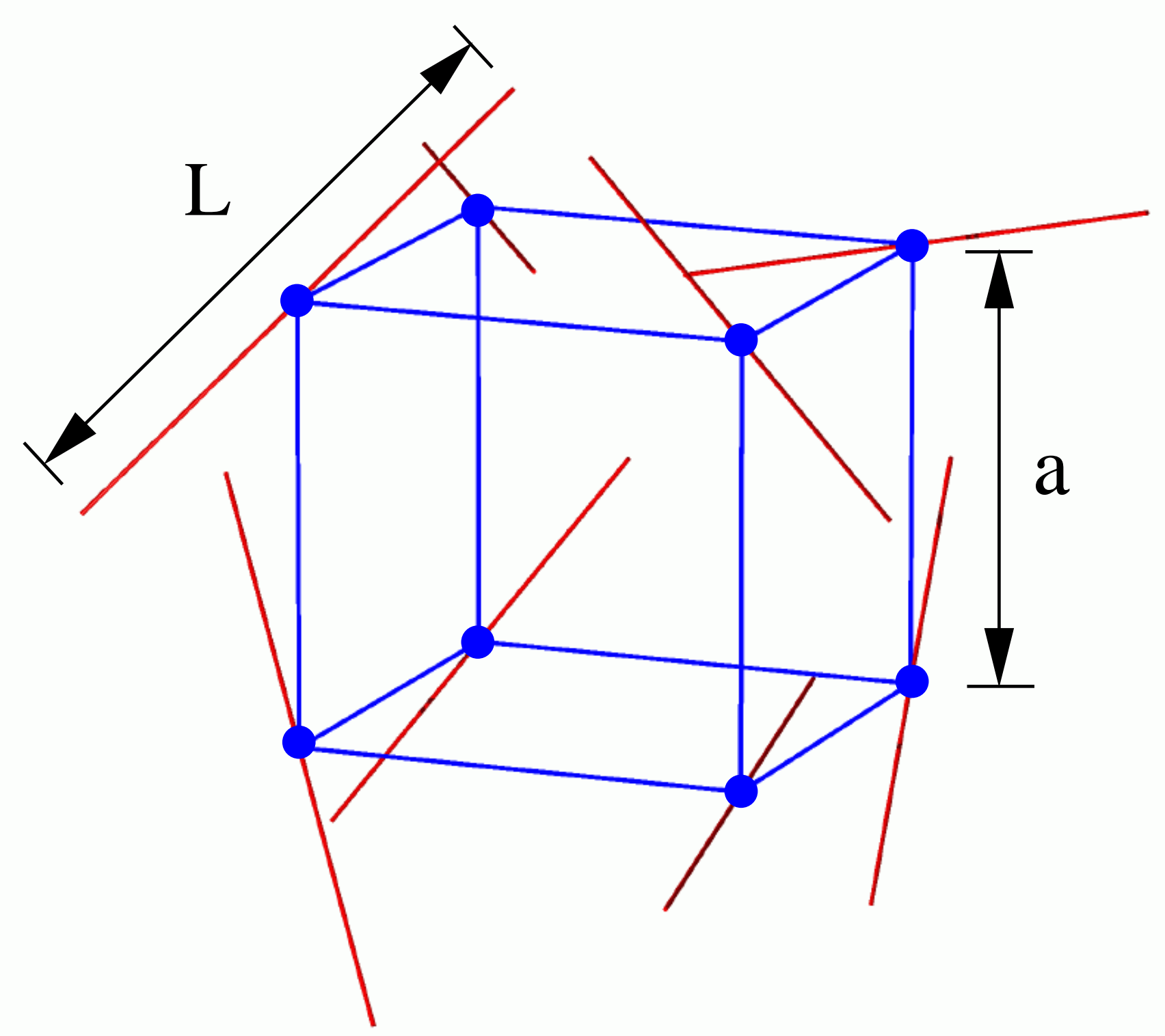




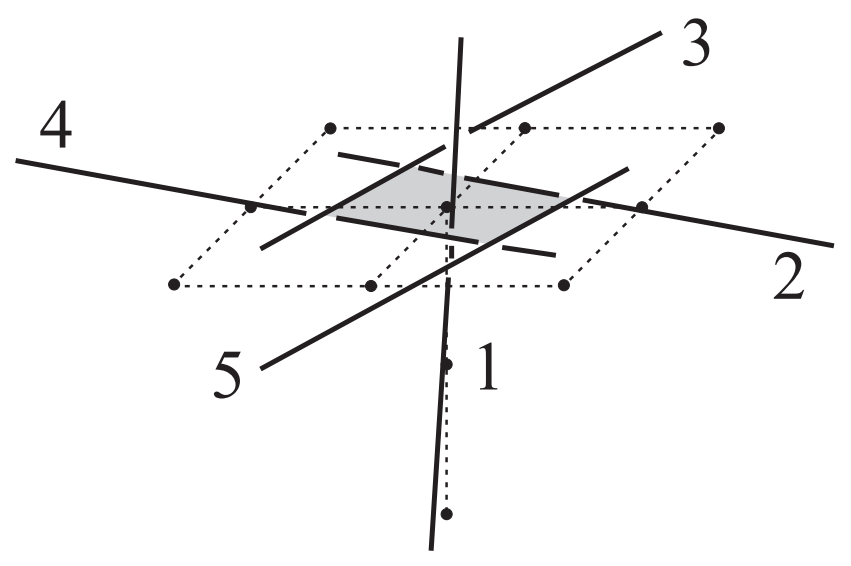




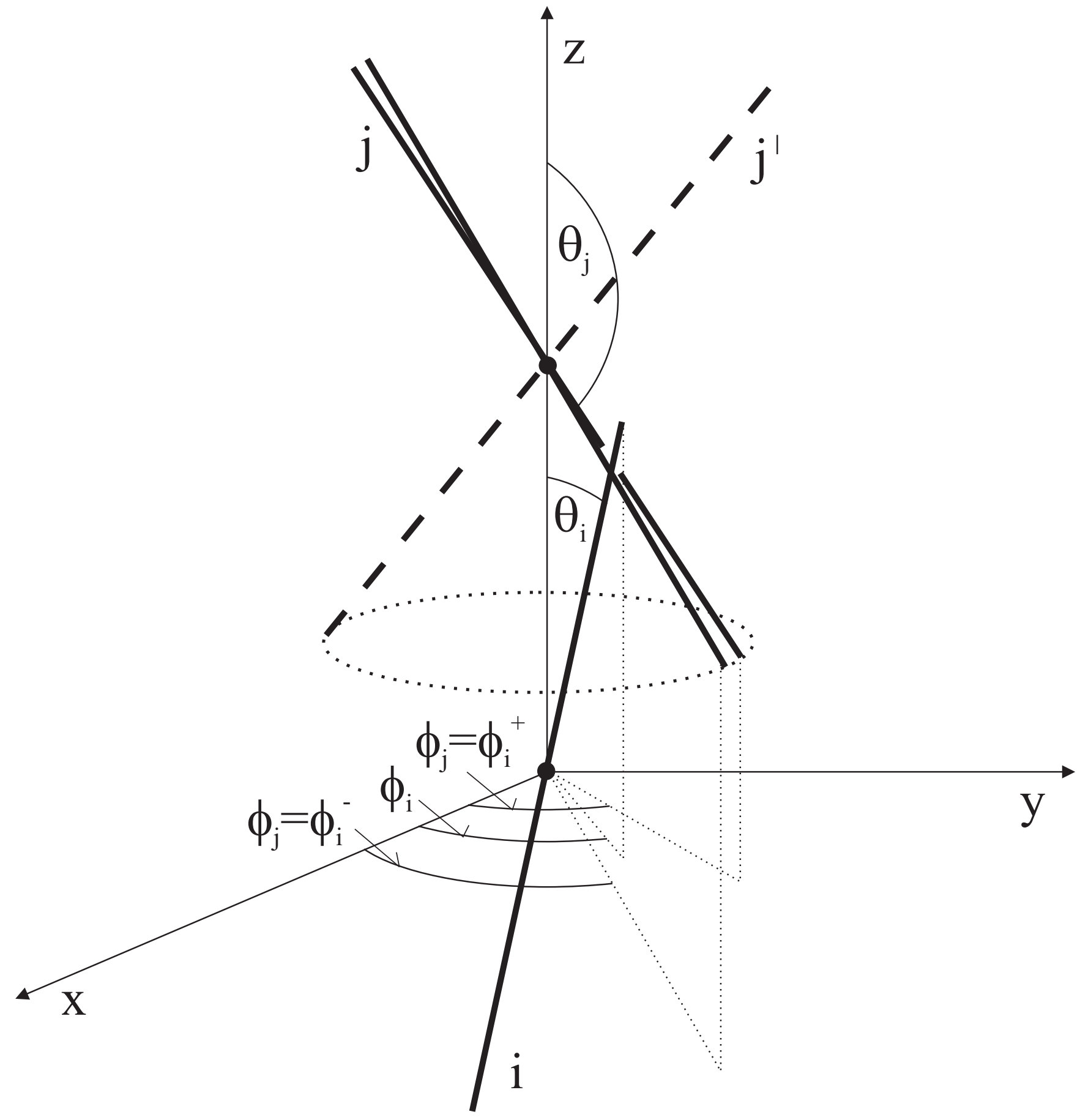




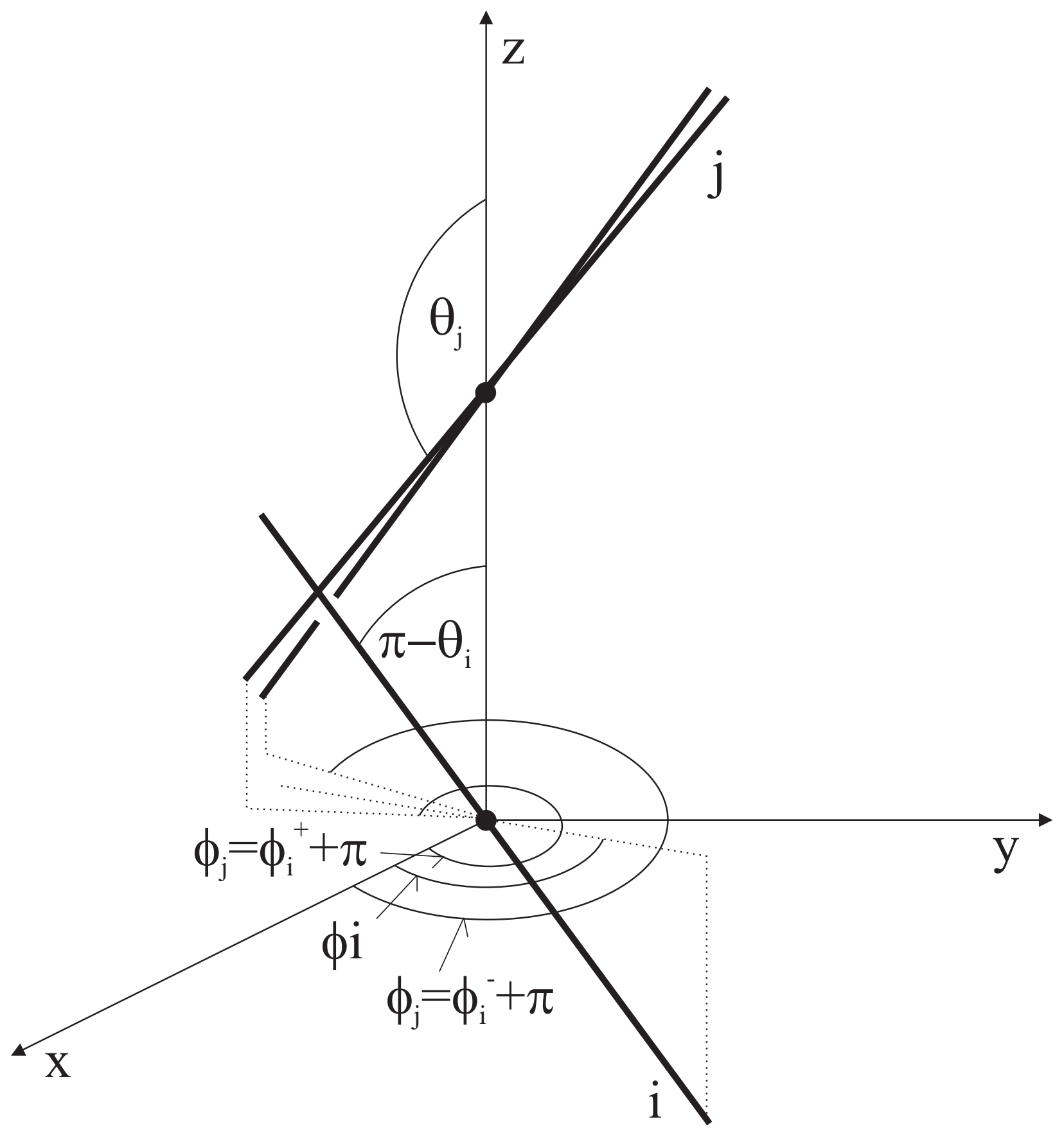




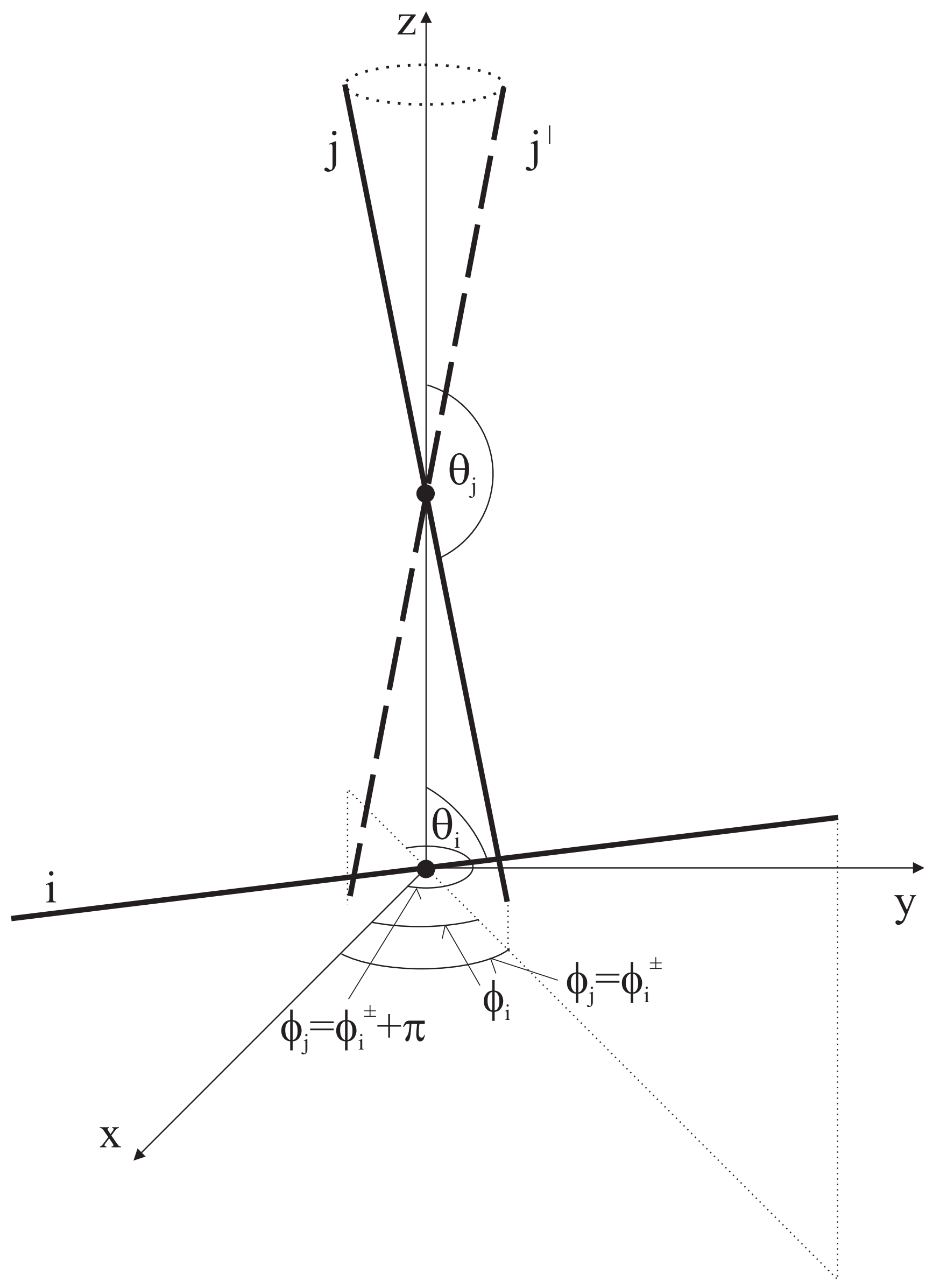




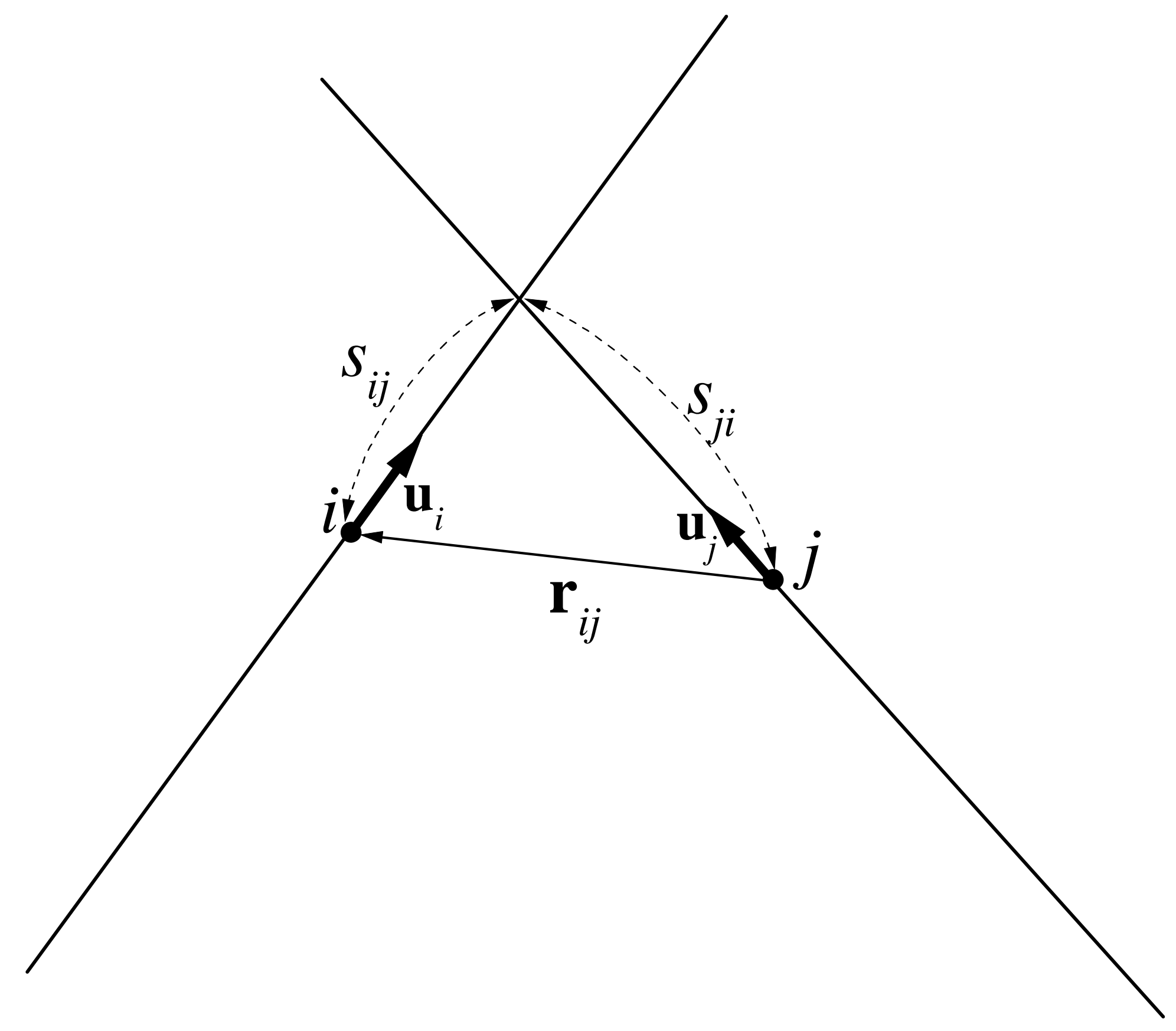




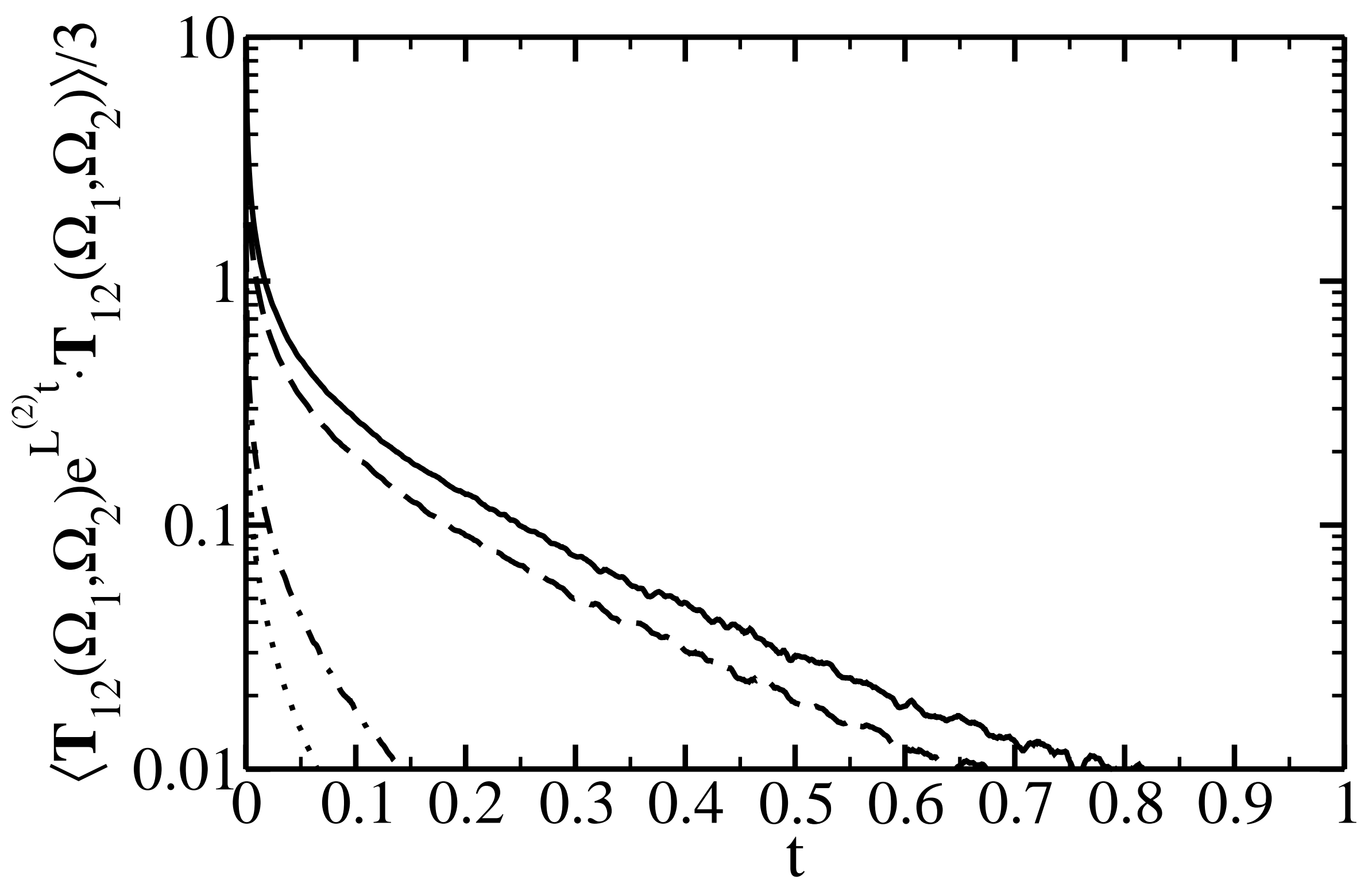




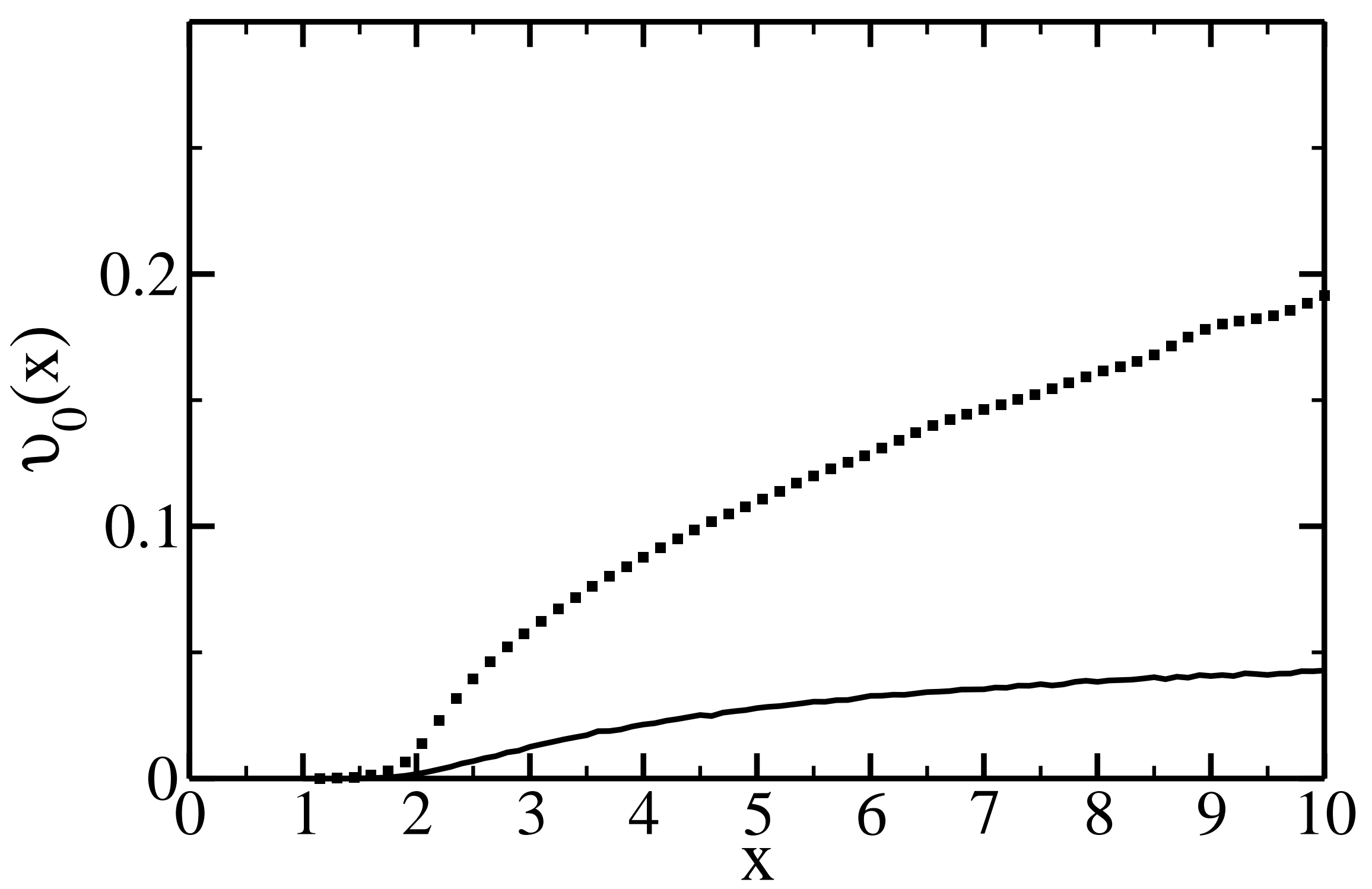




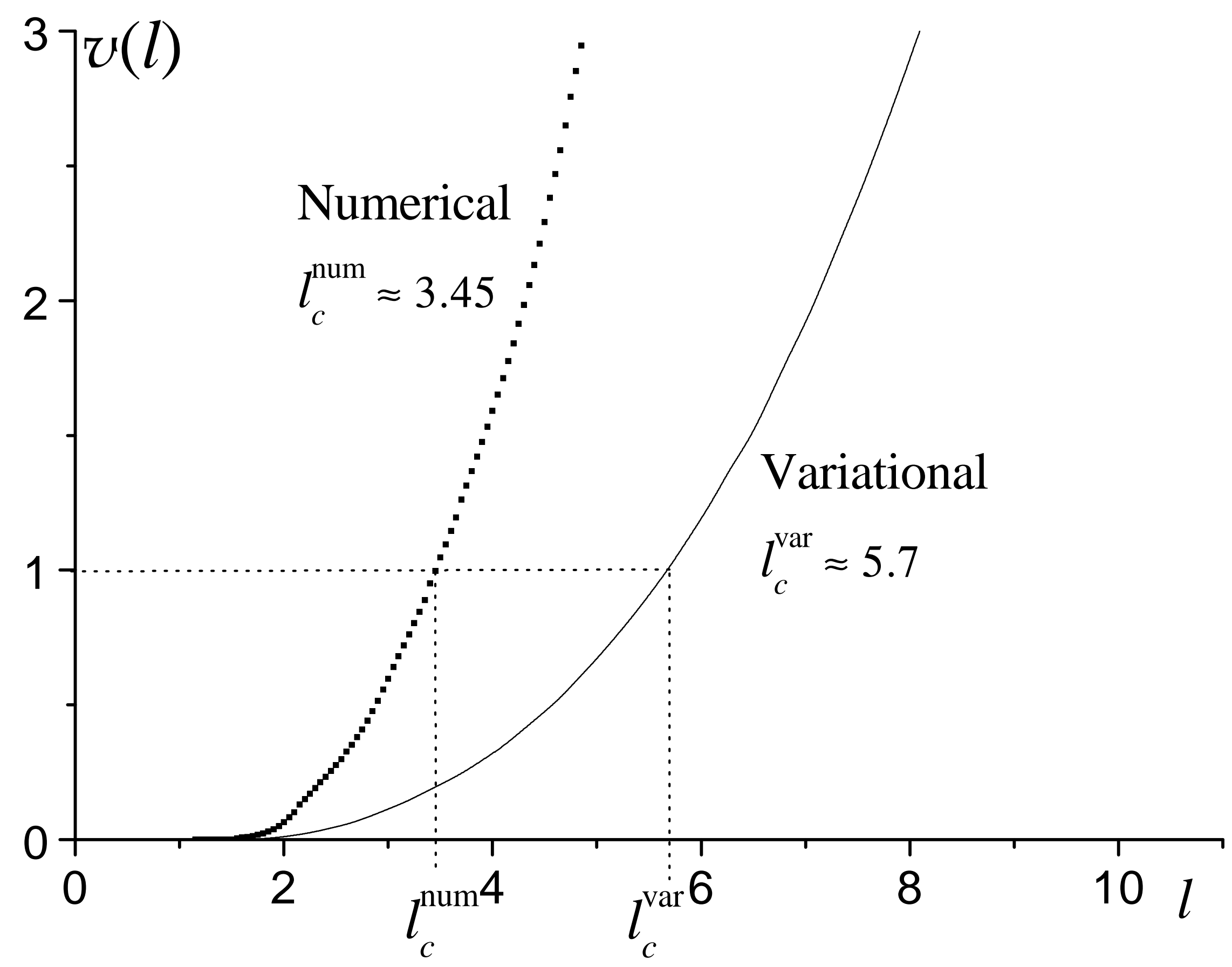

